\title{
The Generalized Covering Salesman Problem
}

\author{
Bruce Golden \\ Robert H. Smith School of Business, University of Maryland, College Park, MD 20742, USA. \\ bgolden@rhsmith.umd.edu \\ Zahra Naji-Azimi \\ DEIS, University of Bologna, Viale Risorgimento 2, 40136 Bologna, Italy. \\ zahra.najiazimi2@unibo.it \\ S. Raghavan \\ Robert H. Smith School of Business, University of Maryland, College Park, MD 20742, USA. \\ raghavan@umd.edu \\ Majid Salari, Paolo Toth \\ DEIS, University of Bologna, Viale Risorgimento 2, 40136 Bologna, Italy. \\ majid.salari2@unibo.it,paolo.toth@unibo.it
}

\begin{abstract}
:
Given a graph $G=(N, E)$, the Covering Salesman Problem (CSP) is to identify the minimum length tour "covering" all the nodes. More specifically, it seeks the minimum length tour visiting a subset of the nodes in $N$ such that each node $i$ not on the tour is within a predetermined distance $d_{i}$ of a node on the tour. In this paper, we define and develop a generalized version of the CSP, and refer to it as the Generalized Covering Salesman Problem (GCSP). Here, each node $i$ needs to be covered at least $k_{i}$ times and there is a cost associated with visiting each node. We seek a minimum cost tour such that each node $i$ is covered at least $k_{i}$ times by the tour. We define three variants of the GCSP. In the first case, each node can be visited by the tour at most once. In the second version, visiting a node $i$ more than once is possible, but an overnight stay is not allowed (i.e., to revisit a node $i$, the tour has to visit another node before it can return to i). Finally, in the third variant, the tour can visit each node more than once consecutively. In this paper, we develop two local search heuristics to find high-quality solutions to the three GCSP variants. In order to test the proposed algorithms, we generated datasets based on TSP Library instances. Since the CSP and the Generalized Traveling Salesman Problem are special cases of the GCSP, we tested our heuristics on both of these two problems as well. Overall, the results show that our proposed heuristics find highquality solutions very rapidly.
\end{abstract}

Key Words: Covering Salesman Problem, Generalized Covering Salesman Problem, Generalized Traveling Salesman Problem, Heuristic Algorithms, Local Search. 


\section{Introduction}

The Traveling Salesman Problem (TSP) is one of the most celebrated combinatorial optimization problems. Given a graph $G=(N, E)$, the goal is to find the minimum length tour of the nodes in $N$, such that the salesman, starting from a node, visits each node exactly once and returns to the starting node (see Dantzig et al., 1954). In recent years, many new variants such as the TSP with profits (Feillet et al., 2005), the Clustered TSP (Chisman, 1975), the Generalized TSP (Fischetti et al., 1997), the Prize Collecting TSP (Fischetti and Toth, 1988), and the Selective TSP (Laporte and Martello, 1990) have been introduced and studied. The recent monograph by Gutin and Punnen (2002) has a nice discussion of different variations of the TSP and solution procedures.

Current (1981) defined and introduced a variant of the TSP called the Covering Salesman Problem (CSP). In the CSP, the goal is to find a minimum length tour of a subset of $n$ given nodes, such that every node $i$ not on the tour is within a predefined covering distance $d_{i}$ of a node on the tour. If $d_{i}=0$ or $d_{i}<\min _{j} c_{i j}$, where $c_{i j}$ denotes the shortest distance between nodes $i$ and $j$, the CSP reduces to a TSP (thus, it is NP-hard). Current and Schilling (1989) referred to several real-world examples, such as routing of rural healthcare delivery teams where the assumption of visiting each city is not required since it is sufficient that each city is near to a stop on the tour (the inhabitants of those cities which are not on the tour are expected to go to their nearest stop). Current and Schilling (1989) also suggested a heuristic for the CSP where in the first step a Set Covering Problem (SCP) over the given nodes is solved. Specifically, to solve the Set Covering Problem, a zero-one $n \times n$ matrix $A$ in which the rows and columns correspond to the nodes is considered. If node $i$ can be covered by node $j$ (i.e., $d_{i} \geq c_{i j}$ ), then $a_{i j}$ is equal to 1 , otherwise it is 0 . Since the value of covering distance $d_{i}$ varies for each node $i$, it should be clear that $A$ is not a symmetric matrix, but for each node $i$ we have $a_{i i}=1$. We should also mention that, in the CSP, there is no cost associated with the nodes, so the column costs of matrix $A$ are all equal to one. Therefore, a unit cost Set Covering Problem is solved in the first step of this algorithm to obtain the cities visited on the tour. Then the algorithm finds the optimal TSP tour over these cities. Since there might be multiple optimal solutions to the SCP, Current and Schilling suggest that all optimal solutions to the SCP be tried out and the best solution be selected. The algorithm is demonstrated on a sample problem, but no additional computational results are reported.

Arkin and Hassin (1994) introduced a geometric version of the Covering Salesman Problem. In this problem, each node specifies a compact set in the plane, its neighborhood, within which the salesman should meet the stop. The goal is computing the shortest length tour that intersects all of the neighborhoods and returns to the initial node. In fact, this problem generalizes the Euclidean 
Traveling Salesman Problem in which the neighborhoods are single points. Unlike the CSP, in which each node $i$ should be within a covering distance $d_{i}$ of the nodes which are visited by the tour, in the geometric version it is sufficient for the tour to intersect the specific neighborhoods without visiting any specific node of the problem. Arkin and Hassin (1994) presented simple heuristics for constructing tours for a variety of neighborhood types. They show that the heuristics provide solutions where the length of the tour is guaranteed to be within a constant factor of the length of the optimal tour. Mennell (2009) studied a special case of the above problem known as the close-enough traveling salesman problem. More specifically, if a salesman gets to within a specified distance (say, $r$ units) of a node, then the node is considered to have been visited. Mennell implemented and compared a number of geometry-based heuristics. Several of these are very fast and produce high quality solutions.

Other than Current (1981), Current and Schilling (1989), and Arkin and Hassin (1994), the CSP does not seem to have received much attention in the literature. However, some generalizations of the CSP have appeared in the literature. One generalization and closely related problem discussed in Gendreau et al. (1997) is the Covering Tour Problem (CTP). Here, some subset of the nodes must be on the tour while the remaining nodes need not be on the tour. Like the CSP, a node $i$ not on the tour must be within a predefined covering distance $d_{i}$ of a node on the tour. When the subset of nodes that must be on the tour is empty the CTP reduces to the CSP, and when the subset of nodes that must be on the tour consists of the entire node set, the CTP reduces to the TSP. Gendreau et al. (1997) proposed a heuristic that combines GENIUS, a high quality heuristic for the TSP (Gendreau et al., 1992), with PRIMAL1, a high quality heuristic for the SCP (Balas and Ho, 1980).

Vogt et al. (2007) considered the Single Vehicle Routing Allocation Problem (SVRAP) that further generalizes the CTP. Here, in addition to tour (routing) costs, nodes covered by the tour (that are not on it) incur an allocation cost, and nodes not covered by the tour incur a penalty cost. If the penalty costs are set high and the allocation costs are set to 0, the SVRAP reduces to the CTP. Vogt et al. (2007) discussed a tabu search algorithm for the SVRAP that includes aspiration, path relinking, and frequency based-diversification.

All of the earlier generalizations of the CSP assume that when a node is covered, its entire demand can be covered. However, in many real-world applications this is not necessarily the case. As an example, suppose we have a concert tour which must visit or cover several cities. Since each show has a limited number of tickets, and large metropolitan areas are likely to have ticket demand which exceeds ticket supply for a single concert, there must be concerts on several nights in each large city in order to fulfill the ticket demand. Also, in the rural healthcare delivery problem, 
discussed in Current and Schilling (1989), when we create a route for the rural medical team, on each day a limited number of people can benefit from the services, so the team should visit some places more than once. Consequently, rather than assuming that a node's demand is completely covered when either it or a node that can cover it is visited, we generalize the CSP by specifying the coverage demand $k_{i}$ which denotes the number of times a node $i$ should be covered. In other words, node $i$ must be covered $k_{i}$ times by a combination of visits to node $i$ and visits to nodes that can cover node $i$. If $k_{i}=1$ for all nodes, we obtain the CSP. This generalization significantly complicates the problem, and is quite different from the earlier generalizations that effectively deal

with unit coverage (i.e., $k_{i}=1$ ). In addition, since in many applications there is a cost for visiting a node (e.g., cost of hotel for staying in a city for one night) we include node visiting costs (for nodes on the tour) in the GCSP. In the next section, we introduce and explain in more detail three different variants that can arise in the GCSP (that deal with whether a node can be revisited or not). All of these variants are strongly NP-Hard, since they contain the classical TSP as a special case.

The rest of this paper is organized as follows. In Section 2, we formally define the Generalized Covering Salesman Problem, and describe three variants. We also present a mathematical model for the problem. Section 3 describes two local search heuristics for the GCSP. Section 4 discusses our computational experience on the three different variants of the GCSP, as well as the CSP and the Generalized TSP (GTSP), which are special cases of the GCSP. Section 5 provides concluding remarks and discusses some possible extensions of the GCSP.

\section{Problem Definition}

In the Generalized Covering Salesman Problem (GCSP), we are given a graph $G=(N, E)$ with $N=\{1,2, \ldots, n\}$ and $E=(\{i, j\}: i, j \in N, i<j)$ as the node and edge sets, respectively. Without loss of generality, we assume the graph is complete with edge lengths satisfying the triangle inequality, and let $c_{i j}$ denote the cost of edge $\{i, j\}$ ( $c_{i j}$ may be simply set to the cost of the shortest path from node $i$ to node $j$ ). Each node $i$ can cover a subset of nodes $D_{i}$ (note that $i \in D_{i}$, and when coverage is based on a covering distance the set $D_{i}$ can be computed easily from the edge costs $C_{i j}$ ) and has a predetermined coverage demand $k_{i} . F_{i}$ is the fixed cost associated with visiting node $i$, and a solution is feasible if each node $i$ is covered at least $k_{i}$ times by the nodes in the tour. The objective is to minimize the total cost, which is the sum of the tour length and the fixed costs associated with the visited nodes. 
We discuss three variants of the GCSP: Binary GCSP, Integer GCSP without overnights, and Integer GCSP with overnights. Next, we explain each of these variants.

Binary Generalized Covering Salesman Problem: In this version, the tour is not allowed to visit a node more than once and after visiting a node we must satisfy the remaining coverage demand of that node by visiting other nodes that can cover it. We use the qualifier binary as this version only permits a node to be visited once.

Integer Generalized Covering Salesman Problem without Overnights: Here, a node can be visited more than once, but an overnight stay is not allowed. Therefore, after visiting a node, the tour can return to this node after having visited at least one other node. In other words, the tour is not allowed to visit a node more than one time consecutively. We use the qualifier integer as this version allows a node to be visited multiple (or an integer number of) times.

Integer Generalized Covering Salesman Problem with Overnights: This version is similar to the previous one, but one or more overnight stays at a node are allowed.

In the CSP, $k_{i}=1$ for all nodes $i \in N$. Clearly, the CSP is a special case of the binary GCSP. When there are unit demands there is no benefit to revisiting a node, consequently the CSP can also be viewed as a special case of the integer variants of the GCSP. Thus, the CSP is a special case of all three variants of the GCSP. As the TSP is a special case of the CSP, all three GCSP variants are strongly NP-Hard.

We now discuss the issue of feasibility of a given instance of the problem. For the binary GCSP, the problem is feasible if demand is covered when all nodes in the graph are visited by the tour. In other words if $h_{j}$ denotes the number of nodes that can cover node $j$ (i.e., $h_{j}$ counts each node $i \in N$ for which $j \in D_{i}$ ), then the problem is feasible if $k_{j} \leq h_{j}$. For the integer GCSP with and without overnights, the problem is always feasible, since a tour on all nodes in the graph may be repeated until all demand is covered.

We now formulate the three different variants of the GCSP. We first provide an integer programming formulation for the binary GCSP, and then an integer programming formulation for the integer GCSP. Our models are on directed graphs (for convenience, as they can easily be extended to asymmetric versions of the problem). Hence, we replace the edge set $E$ by an arc set $A$, where each edge $\{i, j\}$ is replaced by two $\operatorname{arcs}(i, j)$ and $(j, i)$ with identical costs. Also, from the problem data we have available 
$a_{i j}= \begin{cases}1 & \text { if node } \mathrm{j} \text { can cover node } \mathrm{i}, \\ 0 & \text { otherwise }\end{cases}$

We introduce the decision variables:

$w_{i}= \begin{cases}1 & \text { if node } i \text { is on the tour } \\ 0 & \text { otherwise }\end{cases}$

and

$x_{i j}= \begin{cases}1 & \text { if } \operatorname{arc}(i, j) \text { is chosen to be in the solution, } \\ 0 & \text { otherwise }\end{cases}$

The integer programming model can now be stated as:

(BinaryGCSP) Min $\sum_{(i, j) \in A} c_{i j} x_{i j}+\sum_{i \in N} F_{i} w_{i}$

subject to:

$$
\begin{aligned}
& \sum_{j:(j, i) \in A} x_{j i}=\sum_{j:(i, j) \in A} x_{i j}=w_{i} \quad \forall i \in N \\
& \sum_{j \in N} a_{i j} w_{j} \geq k_{i} \quad \forall i \in N \\
& \sum_{l \in S} \sum_{k \in N \backslash S} x_{l k}+\sum_{k \in N \backslash S} \sum_{l \in S} x_{k l} \geq 2\left(w_{i}+w_{j}-1\right) \quad S \subset N, 2 \leq|S| \leq n-2, \quad i \in S, j \in N \backslash S \\
& x_{i j} \in\{0,1\} \quad \forall(i, j) \in A \\
& w_{i} \in\{0,1\} \quad \forall i \in N .
\end{aligned}
$$

The objective is to minimize the sum of the tour costs and the node visiting costs. Constraint set (2) ensures that for each on-tour customer, we have one incoming and one outgoing arc. Constraint set (3) specifies that the demand of each node must be covered. Constraint set (4) is a connectivity constraint that ensures that there are no subtours. Note that there are an exponential number of connectivity constraints. Constraints (5) and (6) define the variables as binary.

For the integer GCSP without overnights we introduce two additional variables to represent the number of times a node is visited, and the number of times an arc is traversed in the tour. Let $y_{i}=$ number of times that node $i$ is visited by the tour, and $z_{i j}=$ number of times arc $(i, j)$ is traversed by the tour.

The integer programming model can now be stated as:

(IntegerGCSP) $\quad$ Min $\sum_{(i, j) \in A} c_{i j} z_{i j}+\sum_{i \in N} F_{i} y_{i}$

subject to:

$$
\begin{aligned}
& \sum_{j:(j, i) \in A}^{S} z_{j i}=\sum_{j:(i, j) \in A} z_{i j}=y_{i} \quad \forall i \in N \\
& \sum_{j \in N} a_{i j} y_{j} \geq k_{i} \quad \forall i \in N \\
& y_{i} \leq L w_{i} \quad \forall i \in N \\
& z_{i j} \leq L x_{i j} \quad \forall(i, j) \in A
\end{aligned}
$$




$$
\begin{array}{ll}
\sum_{l \in S} \sum_{k \in N \backslash S} x_{l k}+\sum_{k \in N \backslash S} \sum_{l \in S} x_{k l} \geq 2\left(w_{i}+w_{j}-1\right) & S \subset N, 2 \leq|S| \leq n-2, \quad i \in S, j \in N \backslash S \\
x_{i j} \in\{0,1\}, z_{i j} \in Z^{+} & \forall(i, j) \in A \\
w_{i} \in\{0,1\}, y_{i} \in Z^{+} & \forall i \in N,
\end{array}
$$

where $L$ is a sufficiently large positive value. The objective is to minimize the sum of the tour costs and the node visiting costs. Constraint set (8) ensures that if node $i$ is visited $y_{i}$ times, then we have $y_{i}$ incoming and $y_{i}$ outgoing arcs. Constraint set (9) specifies that the demand of each node must be covered. Constraint sets (10) and (11) are linking constraints, ensuring that $w_{i}$ and $x_{i j}$ are 1 if $y_{i}$ or $z_{i j}$ are greater than 0 (i.e., if a node is visited or an arc is traversed). Note that it suffices to set $L=\max _{i \in N}\left\{k_{i}\right\}$. Constraint set (12) is a connectivity constraint that ensures that there are no subtours. Note again, that there are an exponential number of connectivity constraints. Finally, constraint sets (13) and (14) define the variables as binary and integer as appropriate. For the integer GCSP with overnights, the above integer programming model (IntegerGCSP) is valid if we augment the arc set $A$ with self loops. Specifically, we add to $A$ the $\operatorname{arc}$ set $\{(i, i): i \in N\}$ (or $A=A \cup\{(i, i): i \in N\})$ with $C_{i i}$ the cost of self loop arcs $(i, i)$ set to 0 .

Note that both the binary GCSP and the integer GCSP formulations rely heavily on the integrality of the node variables. Consequently, the LP-relaxations of these models can be quite poor. Further, these models have an exponential number of constraints, implying that this type of model can only be solved in a cutting plane or a branch-and-cut framework. Thus, considerable strengthening of the above formulations is necessary, before they are viable for obtaining exact solutions to the GCSP. We do not pursue this direction in this paper. Rather, we focus on local search algorithms to develop high-quality solutions for the GCSP.

\section{Local Search Algorithms}

In this section we propose two local search solution procedures, and refer to them as LS1 and LS2, respectively. They are designed to be applicable to all variants of GCSP. In both algorithms, we start from a random initial solution. As we discussed in Section 2, assuming that a problem is feasible (which can be checked easily for the binary GCSP), any random order of the $n$ nodes produces a feasible solution for the binary GCSP, and repeating this ordering a number of times until all demand is covered produces a feasible solution for the integer GCSP. We provide an initial solution to our local search heuristics by considering a random initial ordering of the nodes in the graph and repeat this ordering for the integer variants (if necessary) to cover all of the demand. A natural question at this point may be whether an initial solution different from a random initial ordering provides better final solutions. We actually tested two alternate possibilities for an initial 
solution: (i) the TSP tour obtained using the Lin-Kernighan procedure over the $n$ nodes in the instance, and (ii) the TSP tour obtained using the Lin-Kernighan procedure (Lin and Kernighan, 1973) over the nodes obtained by solving the associated SCP problem to optimality (this solution may not be feasible for the binary GCSP). We found that these two alternatives did not dominate the random initial ordering which is significantly faster in generating an initial solution. As a result, we generate an initial solution using a random initial ordering.

A solution is represented by the sequence of nodes in the tour. Thus, for the binary GCSP, no node may be repeated on the tour, while in the integer GCSP, nodes may be repeated on the tour. For the integer GCSP with no overnights, a repeated node may not be next to itself in the sequence, while in the integer GCSP with overnights a repeated node is allowed to be next to itself in the sequence. Thus, $<1,2,3,4,5,8,9>,<1,2,3,4,3,2,8>$, and $<1,1,2,3,3,8>$ represent tour sequences that do not repeat nodes, repeat nodes but not consecutively, and repeat nodes consecutively. Observe that if the costs are non-negative, then in the integer GCSP with overnights, there is no benefit to leaving a node and returning to revisit it later.

\subsection{LS1}

LS1 tries to find improvements in a solution $S$ by replacing some nodes of the current tour. It achieves this in a two-step manner. First, LS1 deletes a fixed number of nodes. (The number of nodes removed from the tour is equal to a predefined parameter, Search-magnitude, multiplied by the number of nodes in the current tour. If this number is greater than 1 it is rounded down; otherwise, it is rounded up.) It removes a node $k$ from the current solution $S$ with a probability that is related to the current tour and computed as

$$
P_{k}=C_{k} / \sum_{s \in S} C_{s}
$$

where $C_{k}$ is the amount of decrease in the tour cost by deleting node $k$ from $S$; while keeping the rest of the tour sequence as before. (This weighted randomization allows the search procedure to explore a larger set of the feasible solution space and in our preliminary testing worked much better than choosing the node with the largest value of $C_{k}$ for deletion.) Since the deletion of some nodes from the tour $S$ may result in a tour $S^{\prime}$ that is no longer feasible, LS1 attempts to make the solution feasible by inserting new nodes into $S^{\prime}$. We refer to this as the Feasibility Procedure. Suppose that

$P$ is the set of nodes that can be added to the current tour. For the binary GCSP, $P$ consists of the nodes not in the tour $S^{\prime}$, while in the integer GCSP, $P$ consists of all nodes that do not appear more than $L$ times in $S^{\prime}$. We select the node $k \in P$ for which

$$
I_{k} / N_{k}^{2}=\min _{j \in P}\left(I_{j} / N_{j}^{2}\right) .
$$


Here, $I_{k}$ is the amount of increase in the tour cost obtained by inserting node $k$ into its best position in the tour, and $N_{k}$ is the number of uncovered nodes (or uncovered demand) which can be covered by node $k$. (A node that can cover a larger number of uncovered nodes is desirable because it reduces the length of the tour as well as reduces the fixed costs associated with visiting the nodes. We found that squaring $N_{k}$ strengthens this impact in the selection.) We update the calculation of $N_{k}$ for all nodes in $P$ and repeat the selection and insertion of nodes until we obtain a feasible solution. After this step, LS1 checks for the possible removal of "redundant" nodes from the current tour in the Delete_Redundant_Nodes Procedure. A node is redundant if, by removing it, the solution remains feasible.

Next, in case LS1 finds an improvement, i.e., the cost of $S^{\prime}$ is less than the cost of $S$, we would like to try and improve the tour length (and, thus, the overall cost) by applying the LinKernighan Procedure to the solution $S^{\prime}$. Since this procedure is computationally expensive, we only apply it after max $k$ (a parameter) improvements over the solution $S$. We use the Lin-Kernighan code LKH version 1.3 of Helsgaun (2000) that is available for download on the web.

In order to escape from locally optimum solutions, and to search through a larger set in the feasible solution space, we apply a Mutation Procedure whenever the algorithm is not able to increase the quality of the solution after a given number of consecutive iterations. In the mutation procedure, a node is selected randomly and, if the node does not belong to the solution, it is added to the solution in its best place (i.e., the place which causes the minimum increase in the tour length); otherwise, it is removed from the solution. In the latter case, the algorithm calls the feasibility procedure to ensure the solution is feasible, and updates the best solution if necessary.

To add to the diversity within the search procedure, we allow uphill moves with respect to the best solution that LS1 has found. In other words, if the cost of the solution $S$ ' that LS1 obtains is less than $(1+\alpha)$ times the best solution found, we keep it as the current solution (over which we try to find an improvement). Otherwise, we use the best solution obtained so far as the current solution. The stopping criterion for LS1 is a given number of iterations that we denote by max_iter. The pseudo-code of LS1 is given in Algorithm 1. The parameters to be tuned for LS1 and their best values obtained in our computational testing are described in Table 1 (see Section 4).

\subsection{LS2}

This local search procedure tries to improve the cost of a solution by either deleting a node on the tour if the resulting solution is feasible or by extracting a node and substituting it with a 
Algorithm 1: Local Search Algorithm 1 (LS1) for GCSP

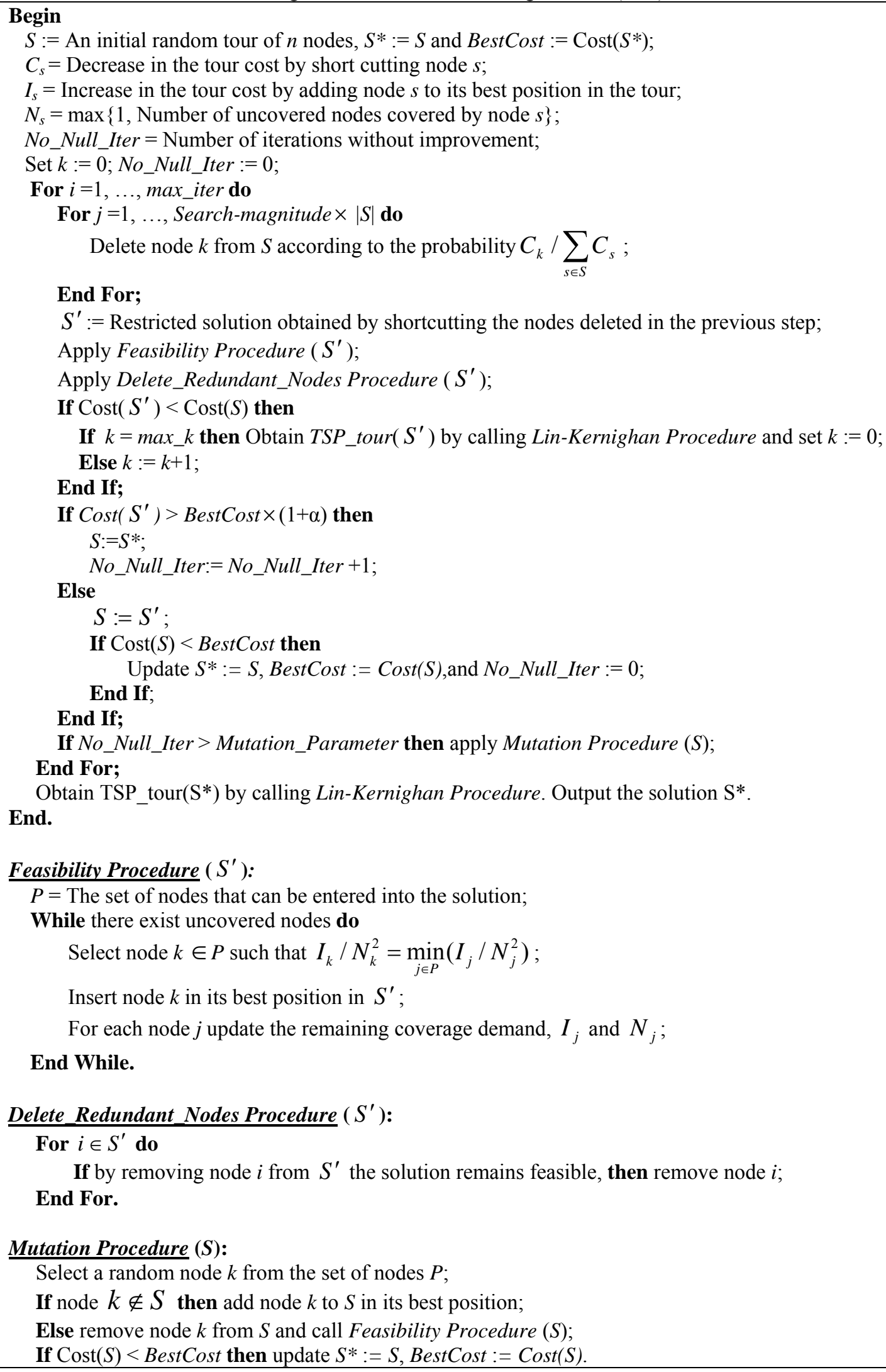

\section{Feasibility Procedure ( $S^{\prime}$ ):}

$P=$ The set of nodes that can be entered into the solution;

While there exist uncovered nodes do

Select node $k \in P$ such that $I_{k} / N_{k}^{2}=\min _{j \in P}\left(I_{j} / N_{j}^{2}\right)$;

Insert node $k$ in its best position in $S^{\prime}$;

For each node $j$ update the remaining coverage demand, $I_{j}$ and $N_{j}$;

End While.

\section{Delete Redundant Nodes Procedure $\left(S^{\prime}\right)$ :}

\section{For $i \in S^{\prime}$ do}

If by removing node $i$ from $S^{\prime}$ the solution remains feasible, then remove node $i$; End For.

\section{Mutation Procedure (S):}

Select a random node $k$ from the set of nodes $P$;

If node $k \notin S$ then add node $k$ to $S$ in its best position;

Else remove node $k$ from $S$ and call Feasibility Procedure $(S)$;

If $\operatorname{Cost}(S)<$ BestCost then update $S^{*}:=S$, BestCost $:=\operatorname{Cost}(S)$. 
promising sequence of nodes. In contrast to LS1, this local search algorithm maintains feasibility (i.e., it only considers feasible neighbors in the local search neighborhood).

LS2 mainly consists of two iterative procedures: the Improvement Procedure and the Perturbation Procedure. In the Improvement Procedure, the algorithm considers extraction of nodes from the current tour in a round-robin fashion. (In other words, given some ordering of nodes on the tour, it first tries to delete the first node on the tour, and then it tries to delete the second node on the tour, and so on, until it tries to delete the last node on the tour.) If by removing a node on the tour the solution remains feasible, the tour cost has improved and the node is deleted from the tour. On the other hand, extracting a node from the tour may cause some other nodes to no longer be fully covered and the solution becomes infeasible. Consequently, in such cases, we try to obtain a feasible solution by substituting the deleted node with a new subsequence of nodes. To this aim, the algorithm considers the $T$ nodes nearest to the extracted node and generates all the promising subsequences with cardinality one or two. Then, it selects the subsequence $s$ that has the minimum insertion cost (i.e., the cost of the tour generated by substituting the deleted node by subsequence $s$ minus the cost of tour with the deleted node). In the case of improvement in the tour cost, we make this substitution; otherwise, we disregard it (i.e., reinsert the deleted node back into its initial position) and continue. The improvement procedure is repeated until it cannot find any improvements (i.e., no change is found while extracting nodes from the current tour in a roundrobin fashion). At the end of the improvement procedure, we apply the 2-Opt procedure to the current tour. (We prefer 2-Opt to the Lin-Kernighan procedure here because it is significantly less expensive computationally.)

In the Perturbation Procedure, LS2 tries to escape from a locally optimum solution by perturbing the solution. In the perturbation procedure, we iteratively add up to $K$ nodes to the tour. It randomly selects one node from among the nodes eligible for addition to the tour (in the binary GCSP, the nodes must be selected from those not in the current tour, while, for the two other GCSP variants, the nodes can be selected from those visited in the current tour also) and inserts it in the tour in its best possible position. Since the tour is feasible prior to the addition of these nodes, the tour remains feasible upon addition of these $K$ nodes.

In one iteration of the procedure, the improvement phase and perturbation phase are iteratively applied $J$ times. After one iteration, when the best solution has improved (i.e., an iteration found a solution with lower cost), we use the Lin-Kernighan Procedure to improve the current tour length (and, thus, the cost of the solution). The stopping criterion for LS2 is a given number of iterations that we denote by max_iter. The pseudo-code for LS2 is given in Algorithm 2, and the parameters to be tuned for LS2 and their best values obtained in our computational testing 
Algorithm 2: Local Search Algorithm 2 (LS2) for the GCSP

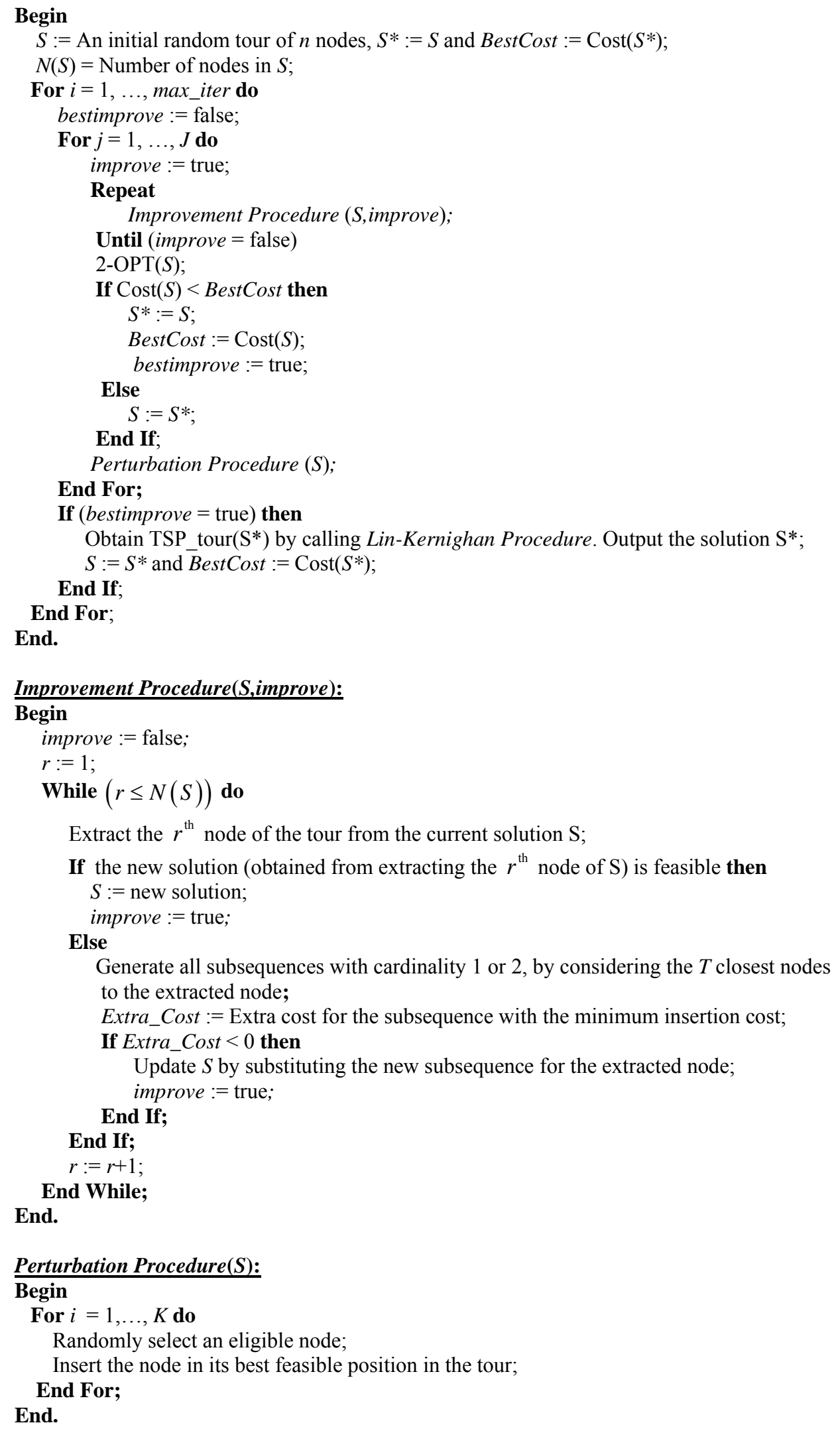


are described in Table 2 (see Section 4).

\section{Computational Experiments}

In this section, we report on our computational experience with the two local search heuristics LS1 and LS2 on the different GCSP variants. We first consider the CSP, and compare the performance of the two proposed heuristics LS1 and LS2, with that of the method proposed by Current and Schilling (1989) for the CSP. Next, we compare LS1 and LS2 on a large number of GCSP instances for the three variants. We also consider a Steiner version of the GCSP, and report our experience with the two local search heuristics. Finally, in order to assess the quality of the solutions found by the two heuristics, we compare them with existing heuristics for the GTSP where there exist well-studied instances in the literature. All of the experiments suggest that the heuristics are of a high quality and run very rapidly.

\subsection{Test Problems}

Since there are no test problems in the literature for the CSP (nor for the variants of the GCSP we introduce), we created datasets based on the TSP library instances (Reinelt, 1991). Our analysis involves two datasets: one consisting of small to medium instances with up to 200 nodes, and the other consisting of large instances with up to 1000 nodes.

The first dataset is constructed based on 16 Euclidean TSPLIB instances whose size ranges from 51 to 200 nodes. In the instances created, each node can cover its 7, 9, or 11 nearest neighbor nodes in addition to itself (resulting in 3 instances for each TSPLIB instance), and each node $i$ must be covered $k_{i}$ times, where $k_{i}$ is a randomly chosen integer number between 1 and 3 . We generated the instances to ensure that a tour over all of the nodes covers the demand (i.e., we ensured that the binary GCSP instances were feasible). Although the cost of visiting a node can be different from node to node, for the first dataset, we consider the node-visiting costs to be the same for all nodes in an instance. In fact, if we assign a large node-visiting cost, the problem becomes a Set Covering Problem (as the node-visiting costs dominate the routing costs) under the assumption that a tour over all the nodes covers the demand. On the other hand, if the node-visiting cost is insignificant (i.e., the routing costs dominate), there is no difference between the integer GCSP with overnights and the CSP. This is because if there is no node-visiting cost, a salesman will stay overnight at a node (at no additional cost) until he/she covers all the demand that can be covered from that node. After testing different values for the node-visiting cost, to ensure that its effect was not at either extreme (Set Covering Problem or CSP), we fixed the node-visiting cost value to 50 for 
Table 1: Parameters for LS1

\begin{tabular}{|c|c|c|}
\hline Parameters & Different values tested & Best value \\
\hline Search-magnitude & $\{0.1,0.2,0.3,0.4,0.5,0.6\}$ & 0.3 \\
\hline Mutation_parameter & $\{5,10,15,20\}$ & 15 \\
\hline max_k & $\{5,10,15,20\}$ & 20 \\
\hline$\alpha$ & $\{0,0.1,0.01,0.001\}$ & 0.1 \\
\hline max_iter & $\{1500,3500,5500,7500,8500\}$ & $\begin{array}{c}3500 \text { (CSP \& Binary GCSP) } \\
7500 \text { (Integer GCSP) }\end{array}$ \\
\hline
\end{tabular}

Table 2: Parameters for LS2

\begin{tabular}{|c|c|c|}
\hline Parameters & Different values tested & Best value \\
\hline$J$ & $\{50,100,150,200,250,300\}$ & 300 \\
\hline$K$ & $\{5,10,15,20\}$ & 10 \\
\hline$T$ & $\{5,10,15\}$ & 10 \\
\hline max_iter & $\{15,20,25,30,35,40,45,50,55,60\}$ & $\begin{array}{c}25 \text { (CSP \& Binary GCSP) } \\
50 \text { (IntegerGCSP) }\end{array}$ \\
\hline
\end{tabular}

the instances in this first dataset. In this fashion, we constructed 48 instances in this first dataset for our computational work.

The second dataset is constructed based on 15 large Euclidean TSPLIB instances whose size ranged from 225 to 1000 nodes. They are constructed in the same fashion as the first dataset, except that (i) each node can cover its 10 nearest neighbor nodes, and (ii) the node-visiting costs are different for the nodes. The node-visiting costs are set randomly in three different ranges. If TL denotes the total length of the solution (using LS2) to the instance as a CSP problem, the nodevisiting costs are set in the following three ranges (resulting in 3 instances for each TSPLIB instance), where (int) indicates we round down to the nearest integer.
A: (int) (Random value in $[0.01,0.05]) *$ TL.
$\mathrm{B}$ : (int) (Random value in $[0.001,0.005]) * \mathrm{TL}$.
C: (int) (Random value in $[0.0001,0.0005]) *$ TL.

Tables 1 and 2 show the different values that were tested for various parameters and the best value obtained for the parameters in LS1 and LS2. To obtain these best parameter values we tested all possible combinations for the parameter values on a set of small test instances consisting of five selected TSPLIB instances (Pr107, Pr124, Pr136, Pr144, and Pr152) for each of the four problems (CSP, binary GCSP, integer GCSP without overnights, and integer GCSP with overnights) where each node can cover its 10 nearest neighbor nodes. These five instances (with a total of 20 instances over the four problems) comprise our training set while our testing set will consist of the instances in the two datasets. This approach is consistent with the one widely used in the data mining and machine learning literature.

Both LS1 and LS2 were implemented in C and tested on a Windows Vista PC with an Intel Core Duo processor running at $1.66 \mathrm{GHz}$ with $1 \mathrm{~GB}$ RAM. As is customary in testing the 
performance of randomized heuristic algorithms, we performed several independent executions of the algorithms. In particular, for each benchmark instance, 5 independent runs of the algorithms LS1 and LS2 were performed, with 5 different seeds for initializing the random number generator and the best and the average performances of the two heuristics are provided.

In all tables reporting the computational performance of the heuristics, the first column is related to the instance name which includes the number of nodes. The second column (NC) gives the number of nearest neighbor nodes that can be covered by each node. Moreover, for each method, the best and the average cost, the number of nodes in the best solution (NB), the average time to best solution (Avg.TB), i.e., the average time until the best solution is found (note the local search heuristic typically continues after this point until it reaches its termination criterion), and the average total time (Avg.TT) are reported (TT is the total time for one run of the local search heuristic). In all tables, in each row the best solution is written in bold and the last two rows give the average of each column (Avg) and the number of best solutions found by each method (No.Best), respectively. All the computing times are expressed in seconds.

\subsection{Comparison of LS1, LS2, and Current and Schilling's Heuristic for the CSP}

Since Current and Schilling (1989) introduced the CSP and proposed a heuristic for it, we compare the performance of LS1 and LS2 against their heuristic. Recall, their algorithm was described in Section 1. Since there are no test instances or computational experiments reported in Current and Schilling's paper, we coded their algorithm to compare the performance of the heuristics. For Current and Schilling's method, we used CPLEX 11 (2007) to generate all optimal solutions of the SCP, and since solving the TSP to optimality is computationally quite expensive on these instances, we use the Lin-Kernighan Procedure to find a TSP tour for each solution. Sometimes, finding all the optimal solutions of an SCP instance is quite time consuming, so we only consider those optimal solutions for the SCP that CPLEX can find in less than 10 minutes of running time.

Table 3 reports the results obtained by LS1, LS2, and our implementation of Current and Schilling's method. In this table, the number of optimal solutions (NO) of the set covering problem is given. In Table 3, instances for which all the optimal solutions to the set covering problem cannot be obtained within the given time threshold are shown with an asterisk. As can be seen in Table 3 for the CSP, both LS1 and LS2 can obtain, in a few seconds, better solutions than Current and Schilling's method. In every case but one (where there is a tie), LS1 and LS2 outperform Current and Schilling's method, while they are several orders of magnitude faster than Current and Schilling's method. Between LS1 and LS2, LS2 outperforms LS1 as it obtains the best solution in 
Figure 1. An example of decreasing the tour length by increasing the number of nodes in Rat99 $(\mathrm{NC}=7)$.

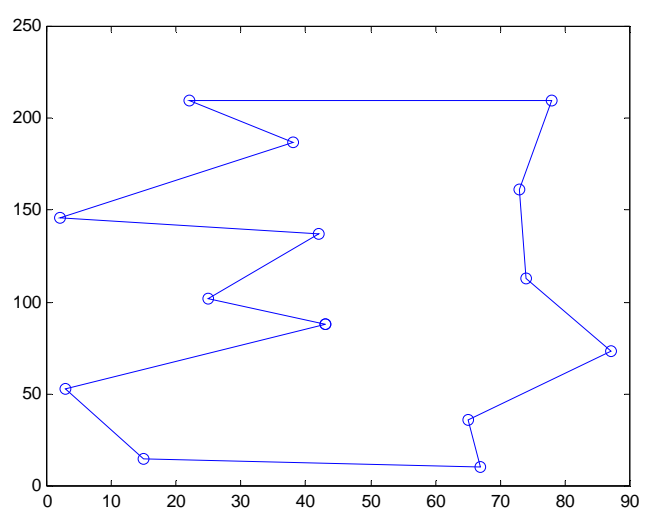

a) Number of nodes in the tour: 14, Tour length: 572

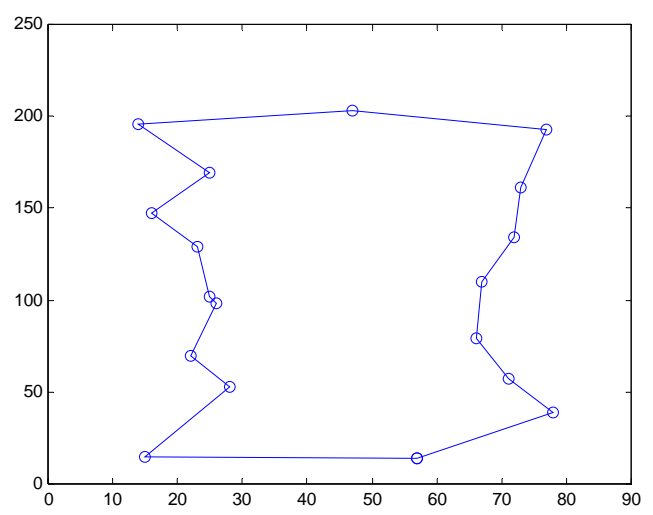

b) Number of nodes in the tour: 18, Tour length: 486

Figure 2. An example of decreasing the tour length by increasing the number of nodes in KroA200 (NC=7).

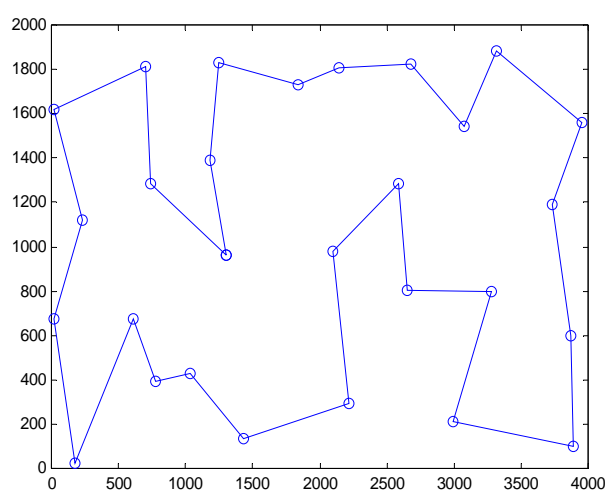

a) Number of nodes in the tour: 28, Tour length: 14667

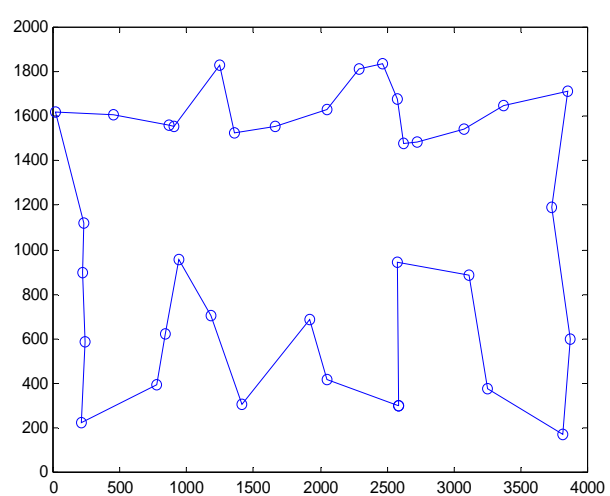

b) Number of nodes in the tour: 34, Tour length: 13285

all 48 instances, while LS1 only obtains the best solution in 39 out of the 48 instances. We applied the Wilcoxon signed rank test (Wilcoxon, 1945), a well-known nonparametric statistical test to perform a pairwise comparison of the heuristics. In each comparison, the null hypothesis is that the two heuristics being compared are equivalent in performance (with the alternate hypothesis that one of them is superior). Based on this analysis we found that LS1 outperforms Current and Schilling's heuristic at a significance level of $\alpha<0.0001$ (in other words, we can reject the null hypothesis that the performance of LS1 and Current and Schilling's heuristic are equivalent at a significance level of $\alpha<0.0001$ and accept the alternate hypothesis that LS1 outperforms Current and Schilling's heuristic). We also found that LS2 outperforms Current and Schilling's heuristic at a significance level of $\alpha<0.0001$. When we compared LS2 and LS1, we found that LS2 outperforms LS1 at a significance level of $\alpha=0.002$.

From Table 3 we can make the following observation. Sometimes by selecting a larger number of nodes to visit, we are able to find a shorter tour length, so the optimal solution of the Set 
Covering Problem is not necessary a good solution for the Covering Salesman Problem. This is contrary to the intuition at the heart of Current and Schilling's method. Figures 1 and 2 illustrate two examples of CSP (Rat99 and KroA200) in which, by increasing the number of nodes in the tour, the tour length is decreased.

\subsection{Comparison of LS1 and LS2 on GCSP Variants}

In Table 4 the results of the two local search heuristics on the binary GCSP are given. As can be seen in this table, for the binary GCSP, the two local search heuristics are very competitive with each other. On average, LS1 is a bit faster than LS2. In terms of the average cost, the number of best solutions found, and average time, LS1 is slightly better than LS2. However, LS2 is faster in terms of the average time to best solution. Over the 48 instances, the two heuristics were tied in 21 instances. In 14 instances, LS1 is strictly better than LS2, and, in 13 instances, LS2 is strictly better than LS1. When we compared LS1 and LS2 using the Wilcoxon signed-rank test, we found that the difference between the two was not statistically significant. (Here $\alpha=0.1469$, and we used a $5 \%$ significance level for the hypothesis tests. In other words we reject the null hypothesis that the performance of the heuristics is equal only if $\alpha<0.05$ ).

Table 5 provides a comparison of LS1 and LS2 on the integer GCSP without overnights. Here, the table contains one additional column reporting the number of times a solution revisits cities (NR). Here, over 48 test instances, LS1 is strictly better than LS2 in 15 instances, LS2 is strictly better than LS1 in 8 instances, while they are tied in 25 instances. Again, the running times of both LS1 and LS2 are generally small, taking no more than 32 seconds, even for the largest instances. When we compared LS1 and LS2 using the Wilcoxon signed rank test, we found that the difference between the two was not statistically significant (here $\alpha=0.1264$ ).

Table 6 compares LS1 and LS2 on integer GCSP with overnights. Here, the table contains one additional column reporting the number of times a solution stays overnight at a node (ON). Over 48 test instances, LS1 is strictly better than LS2 in 9 instances, LS2 is strictly better than LS1 in 26 instances, and they are tied in 13 instances. However, the running time of LS1 increases significantly with instance size compared to LS2. This increase in running time appears to be due to a significant increase in the number of times LS1 calls the Lin-Kernighan Procedure. Overall, LS2 appears to be a better choice than LS1 for the integer GCSP with overnights. When we compared LS1 and LS2 using the Wilcoxon signed rank test, we found that LS2 outperforms LS1 at a significance level of $\alpha=0.0027$.

Notice that a solution to the binary GCSP is a feasible solution to the integer GCSP without overnights, and a feasible solution to the integer GCSP without overnights is a feasible solution for 
the integer GCSP with overnights. Hence, we should expect that the average cost of the solutions found should go down as we move from Table 4 to Table 6 . This is confirmed in our experiments.

\subsection{GCSP with Steiner Nodes}

In our earlier test instances, every node had a demand. We now construct some Steiner instances, i.e., ones where some nodes have $k_{i}$ set to zero (the rest of the demands remain unchanged). In these cases, a tour could contain some "Steiner nodes" (i.e., nodes without any demand) that can help satisfy the coverage demand of the surrounding (or nearby) nodes. On the other hand, if fewer nodes have demands then it is likely that fewer nodes need to be visited (in particular, the earlier solutions obtained are feasible for the Steiner versions), and, thus, we would expect the cost of the solutions to the GCSP with Steiner nodes to be lower compared to the instances of the GCSP without Steiner nodes. Table 7 confirms this observation. Here, we compare LS1 and LS2 on the CSP with Steiner nodes. For each CSP instance (in Table 3) we select 10 percent of the nodes randomly and set their corresponding demands to zero. The behavior of LS1 and LS2 is similar to that of the earlier CSP instances. Specifically, over the 48 test instances, LS2 obtains the best solution in all 48 instances while LS1 only obtains the best solution in 40 out of 48 instances. Although LS2 outperforms LS1 in terms of finding better solutions, overall, LS1 runs faster than LS2. When we compared LS1 and LS2 using the Wilcoxon signed rank test, we found that LS2 outperforms LS1 at a significance level of $\alpha=0.0039$. For brevity, we have limited the Steiner nodes comparison to the CSP.

\subsection{Large-Scale Instances}

Tables 8, 9, and 10 report on our experience with the second dataset consisting of the large scale instances with varying node-visiting costs. As the results show, LS2 significantly outperforms LS1. In particular for the binary GCSP, LS2 is strictly better than LS1 in 43 out of 45 instances, while LS1 is strictly better than LS2 in 2 out of 45 instances. The Wilcoxon signed rank test indicates that LS2 outperforms LS1 at a significance level of $\alpha<0.0001$. In terms of average running time, LS2 is an order of magnitude (about 10 times) faster than LS1. For the GCSP without overnights, LS2 is strictly better than LS1 in 44 out of 45 instances, while LS1 is strictly better than LS2 in 1 out of 45 instances. The Wilcoxon signed rank test indicates that LS2 outperforms LS1 at a significance level of $\alpha<0.0001$. Again, in terms of running time, LS2 is an order of magnitude faster than LS1. Even in the largest instances, LS2 takes no more than 4 minutes, while LS1 can take over 20 minutes to run. For the GCSP with overnights, LS2 is strictly better than LS1 in 44 out of 45 instances, while LS1 is strictly better than LS2 in 1 out of 45 instances. The Wilcoxon 
signed rank test indicates that LS2 outperforms LS1 at a significance level of $\alpha<0.0001$. Again, in terms of running time, LS2 is much faster than LS1, taking only about 3 minutes even for the largest 1000 node instance.

Taken together with the results on the first dataset, overall, LS2 seems to be a better choice than LS1. On the first dataset it outperforms LS1 (in a statistically significant sense) on the CSP, the Steiner CSP, and the integer GCSP with overnights. While the difference between LS1 and LS2 for the binary GCSP and the integer GCSP without overnights on the first dataset is not statistically significant. However, the results on the larger instances in the second dataset clearly show the superiority of LS2 over LS1 in terms of performance on all three problems, as well as its robustness in terms of running time.

\subsection{Analyzing the Quality of LS2 on the Generalized TSP}

Since we do not have lower bounds or optimal solutions for the CSP and GCSP instances, it is hard to assess the quality of the solutions. Noting that the generalized TSP (GTSP) is a special case of the CSP (we explain how momentarily), we use some well-studied GTSP instances in the literature (see Fischetti et al., 1997) and compare LS2 with eight different heuristics designed specifically for the GTSP; as well as to the optimal solutions on these instances obtained by Fischetti et al. (1997) using a branch-and-cut method. In the GTSP, the set of nodes in the graph are clustered into disjoint sets and the goal is to find the minimum length tour over a subset of nodes so that at least one node from each cluster is visited by the tour. This can be formulated as a CSP, where each node has unit demand (i.e., $k_{i}=1$ for each node $i$ ) and each node in a cluster covers every other node in a cluster (and no other nodes).

We executed LS2 on the benchmark GTSP dataset (see Fischetti et al., 1997) by first tuning its parameters. The tuned parameters of LS2 are configured as follows: $J=300, K=10, T=10$, and max_iter $=50$ and 10 independent runs of LS2 were performed. We compared LS2 to eight other heuristics in the literature that are listed below.

1. MSA: A Multi-Start Heuristic by Cacchiani et al. 2011,

2. mrOX: a Genetic Algorithm by Silberholz and Golden 2007,

3. RACS: a Reinforcing Ant Colony System by Pintea et al. 2007,

4. GA: a Genetic Algorithm by Snyder and Daskin 2006,

5. $G I^{3}$ : a composite algorithm by Renaud and Boctor 1998 ,

6. NN: a Nearest Neighbor approach by Noon 1988,

7. FST-Lagr and FST-root: Two heuristics by Fischetti et al. 1997. 
In order to perform a fair comparison on the running times of the different heuristics, we scaled the running times for the different computers as indicated in Dongarra (2004). The computer factors are shown in Table 11. The columns indicate the computer used, solution method used, Mflops of the computer, and $r$ the scaling factor. Thus the reported running times in the different papers are appropriately multiplied by the scaling factor $r$. We note that an identical approach was taken in Cacchiani et al. (2011) to compare across these heuristics for the GTSP. Since no computer information is available for the RACS heuristic, we use a scaling factor of 1 .

Table 12 reports on the comparison. For each instance, we report the percentage gap with respect to the optimal solution value and the computing time (expressed in seconds and scaled according to the computer factors given in Table 11) for all the methods except for B\&C (for which we report only the computing time). Some of the methods (RACS, GI ${ }^{3}$, and NN) only reported solutions for 36 of the 41 instances. Consequently, in the last four rows of Table 12, we report for each algorithm, the average percentage gap and the average running time on the 36 instances tested by all the methods, as well as over all 41 instances (for all methods except RACS, GI ${ }^{3}$, and NN). We also summarize the number of times the optimum solution was found by a method. As Table 12 indicates, although LS2 was not explicitly developed for the GTSP (but rather for a generalization of it), it performs quite well. On average, it takes 2.2 seconds, and finds solutions that are $0.08 \%$ from optimality, and it found optimal solutions in 30 out of 41 benchmark GTSP instances.

\section{Summary and Conclusions}

In this paper, we considered the CSP, and introduced a generalization quite different from earlier generalizations of the CSP in the literature. Specifically, in our generalization, nodes must be covered multiple times (i.e., we introduce a notion of coverage demand of a node). This may require a tour to visit a node multiple times (which is not the case in earlier generalizations), and there are also node-visiting costs. We discussed three variants of the GCSP. The binary GCSP where revisiting a node is not permitted, the integer GCSP without overnights where revisiting a node is permitted only after another node is visited, and the integer GCSP with overnights where revisiting a node is permitted without any restrictions. We designed two local search heuristics, LS1 and LS2, for these variants. In a certain sense, LS1 is a very large-scale neighborhood search (VLNS) algorithm (because it relies heavily on the Lin-Kernighan procedure and a neighborhood whose size is variable and large), and LS2 is like an iterated local search (ILS) algorithm (because it applies an improvement and perturbation procedure iteratively and the sophisticated Lin-Kernighan procedure is applied infrequently). Nevertheless, the Lin-Kernighan procedure plays a significant role in the performance of both algorithms. Indeed, if the Lin-Kernighan procedure were replaced 
by the 2-OPT procedure in the algorithms, the performance of the heuristics is significantly worse. Overall, LS2 appears to be superior in terms of its running time as well as its performance with respect to the number of times it found the best solutions for the different variants. When LS2 is compared to 8 benchmark heuristics for the GTSP (that were specifically designed for the GTSP), LS2 performs quite well, finding high-quality solutions rapidly.

We introduced two integer programming models for the binary and integer GCSP, respectively. However, both these models require considerable strengthening and embedding in a branch-and-cut framework in order to obtain exact solutions to the GCSP. This is a natural direction for future research on the GCSP (as it will provide an even better assessment of the quality of heuristics for the GCSP), and we hope researchers will take up this challenge.

Some natural generalizations of the GCSP (along the lines of the earlier generalizations of the CSP) may also be considered in future research. The earlier generalizations of the CSP (see Vogt et al., 2007) included constraints in terms of (i) requiring some nodes to be on the tour, (ii) requiring some nodes not to be on the tour, (iii) allowing a node not to be covered at a cost (for our GCSP that would mean the covering demand of a node could be partially covered at a cost), and (iv) including a cost for allocating nodes not on the tour to the tour. These would be natural generalizations of this multi-unit coverage demand variant of the CSP that we have introduced.

\section{References}

Arkin E.M. and Hassin R. 1994. Approximation Algorithms for the Geometric Covering Salesman Problem, Discrete Applied Mathematics 55(3), 197-218.

Balas E. and Ho A. 1980. Set Covering Algorithms Using Cutting Planes, Heuristics, and Subgradient Optimization: A Computational Study. Math. Programming, 12, 37-60.

Cacchiani V., Fernandes Muritiba A.E., Negreiros M., and Toth P. 2011. A Multi-Start Heuristic For the Equality Generalized Traveling Salesman Problem, Networks, to appear.

Chisman, J. A. 1975. The Clustered Traveling Salesman Problem, Computers and Operations Research, 2(2), 115-119.

Current J.R. 1981. Multi-objective design of Transportation Networks, Ph.D thesis, Department of Geography and Environmental Engineering. The Johns Hopkins University, Baltimore.

Current J.R., and Schilling D.A. 1989. The Covering Salesman Problem. Transportation Science, 23(3), 208213.

Dantzig G.B., Fulkerson R., and Johnson S.M. 1954. Solution of a Large Scale Traveling Salesman Problem. Operations Research, 2(4), 393-410.

Dongarra J.J. 2004, Performance of various computers using standard linear equations software, Technical Report CS-89-85, Computer Science department, University of Tennessee. 
Feillet D., Dejax P., and Gendreau M. 2005. Traveling Salesman Problems with Profits. Transportation Science, 39(2), 188-205.

Fischetti M., and Toth P. 1988. An Additive Approach for the Optimal Solution of the Prize-Collecting Traveling Salesman Problem. In Vehicle Routing: Methods and Studies. Golden B. L. and Assad A. A. (eds.). North-Holland, Amsterdam, 319-343.

Fischetti M., Salazar-Gonzalez J.J., and Toth P. 1997. A Branch and Cut Algorithm for the Symmetric Generalized Traveling Salesman Problem. Operations Research, 45(3), 378-394.

Gendreau M., Hertz A., and Laporte G. 1992. New Insertion and Postoptimization Procedures for the Traveling Salesman Problem. Operations Research, 40(6), 1086-1094.

Gendreau M., Laporte G., and Semet F. 1997. The Covering Tour Problem. Operations Research, 45(4), 568-576.

Gutin G. Punnen A.P. (Eds.). 2002. The Traveling Salesman Problem and Its Variations. Kluwer Academic publishers, Netherlands.

Helsgaun K. 2000. An Effective Implementation of the Lin-Kernighan Traveling Salesman Heuristic. European Journal of Operational Research, 126 (1), 106-130.

ILOG Cplex 11.0, 2007. User's Manual and Reference Manual, ILOG, S.A., http://www.ilog.com.

Laporte G., and Martello S. 1990. The Selective Traveling Salesman Problem. Discrete Applied. Mathematics, 26, 193-207.

Lin S., and Kernighan B.W. 1973. An Effective Heuristic Algorithm for the Traveling Salesman Problem. Operations Research, 21(2), 498-516.

Mennell W. 2009. Heuristics for solving three routing problems: Close enough traveling salesman problem, close-enough vehicle routing problem, sequence-dependent team orienteering problem. Ph.D thesis, University of Maryland, College Park.

Noon C. E. 1988. The generalized traveling salesman problem, Ph.D. thesis, University of Michigan.

Pintea C.M., Pop P.C., and Chira C. 2007. The Generalized Traveling Salesman Problem Solved with Ant Algorithms, Journal of Universal Computer Science, 13(7), 1065-1075.

Reinelt G. 1991. A Traveling Salesman Problem Library. ORSA Journal on Computing, 3(4), 376-384.

Renaud J., and Boctor F.F. 1998. An efficient composite heuristic for the symmetric generalized traveling salesman problem, European Journal of Operational Research, 108(3), 571-584.

Silberholz J. and Golden B. 2007. The Generalized Traveling Salesman Problem: a new Genetic Algorithm approach, in Baker E.K., Joseph A., Mehrotra A., and Trick M.A.(eds.) Extending the Horizons: Advances in Computing, Optimization Decision Technologies, Springer, 165-181.

Snyder L.V., and Daskin M.S. 2006. A random-key genetic algorithm for the generalized traveling salesman problem, European Journal of Operational Research, 174(1), 38-53.

Vogt L., Poojari CA. and Beasley JE. 2007. A Tabu Search algorithm for the Single Vehicle Routing Allocation Problem, Journal of Operational Research Society, 58(4), 467-480.

Wilcoxon, F. 1945. Individual comparisons by ranking methods, Biometrics, 1(6), 80-83. 
Table 3. Comparison of Current and Schilling's method with LS1 and LS2 for CSP

\begin{tabular}{|c|c|c|c|c|c|c|c|c|c|c|c|c|c|c|c|c|}
\hline \multirow[b]{2}{*}{ Instance } & \multirow[b]{2}{*}{$\mathrm{NC}$} & \multicolumn{5}{|c|}{ Current and Schilling } & \multicolumn{5}{|c|}{ LS1 } & \multicolumn{5}{|c|}{ LS2 } \\
\hline & & NO & Cost & NB & $\mathrm{TB}$ & TT & $\begin{array}{l}\text { Best } \\
\text { Cost }\end{array}$ & $\begin{array}{l}\text { Avg. } \\
\text { Cost }\end{array}$ & NB & $\begin{array}{c}\text { Avg. } \\
\text { TB }\end{array}$ & $\begin{array}{c}\text { Avg. } \\
\text { TT }\end{array}$ & $\begin{array}{l}\text { Best } \\
\text { Cost }\end{array}$ & $\begin{array}{l}\text { Avg. } \\
\text { Cost }\end{array}$ & NB & $\begin{array}{c}\text { Avg. } \\
\text { TB }\end{array}$ & $\begin{array}{r}\text { Avg } \\
\text { TT }\end{array}$ \\
\hline \multirow{3}{*}{ Eil 51} & 7 & 13 & 194 & 7 & 0.07 & 0.21 & 164 & 164.0 & 10 & 0.05 & 0.73 & 164 & 164.0 & 10 & 0.04 & 1.05 \\
\hline & 9 & 309 & 169 & 6 & 1.92 & 1.97 & 159 & 159.0 & 8 & 0.16 & 1.51 & 159 & 159.0 & 9 & 0.04 & 0.78 \\
\hline & 11 & 282 & 167 & 5 & 0.59 & 1.70 & 147 & 147.0 & 7 & 0.04 & 0.69 & 147 & 147.0 & 7 & 0.04 & 0.87 \\
\hline \multirow{3}{*}{ Berlin 52} & 7 & 2769 & 4019 & 8 & 19.39 & 21.04 & 3887 & 3887.0 & 12 & 0.42 & 0.73 & 3887 & 3887.0 & 11 & 0.42 & 1.05 \\
\hline & 9 & 11478 & 3430 & 7 & 26.08 & 94.14 & 3430 & 3430.0 & 7 & 0.03 & 1.32 & 3430 & 3430.0 & 7 & 0.04 & 0.95 \\
\hline & 11 & 11 & 3742 & 5 & 0.22 & 0.26 & 3262 & 3262.0 & 6 & 0.02 & 0.74 & 3262 & 3262.0 & 6 & 0.04 & 0.55 \\
\hline \multirow{3}{*}{ St 70} & 7 & 32832 & 297 & 10 & 232.24 & 454.07 & 288 & 288.0 & 11 & 0.08 & 0.87 & 288 & 288.0 & 11 & 0.06 & 1.48 \\
\hline & 9 & 18587 & 271 & 9 & 173.87 & 176.00 & 259 & 259.0 & 10 & 0.03 & 1.45 & 259 & 259.0 & 10 & 0.06 & 1.65 \\
\hline & 11 & 1736 & 269 & 7 & 13.21 & 13.74 & 247 & 247.0 & 10 & 0.12 & 0.83 & 247 & 247.0 & 10 & 0.05 & 1.37 \\
\hline \multirow{3}{*}{ Eil 76} & 7 & 241 & 241 & 11 & 1.15 & 2.46 & 207 & 207.0 & 17 & 0.12 & 0.70 & 207 & 207.0 & 15 & 0.27 & 1.35 \\
\hline & 9 & 1439 & 193 & 9 & 7.43 & 13.95 & 186 & 186.0 & 11 & 0.37 & 1.59 & 185 & 185.0 & 12 & 0.06 & 1.43 \\
\hline & 11 & 7050 & 180 & 8 & 30.48 & 78.88 & 170 & 170.0 & 12 & 0.24 & 0.78 & 170 & 170.0 & 11 & 0.06 & 1.50 \\
\hline \multirow{3}{*}{$\operatorname{Pr} 76$} & 7 & 26710 & 53255 & 11 & 54.20 & 170.41 & 50275 & 50275.0 & 15 & 0.15 & 0.85 & 50275 & 50275.0 & 14 & 0.10 & 1.89 \\
\hline & 9 & $326703 *$ & 45792 & 10 & 6743.66 & 9837.36 & 45348 & 45389.6 & 13 & 0.57 & 2.45 & 45348 & 45462.2 & 12 & 0.06 & 1.50 \\
\hline & 11 & 20 & 45955 & 7 & 0.11 & 0.20 & 43028 & 43028.0 & 11 & 0.29 & 0.69 & 43028 & 43028.0 & 10 & 0.13 & 1.39 \\
\hline \multirow{3}{*}{ Rat 99} & 7 & 3968 & 572 & 14 & 22.74 & 32.75 & 486 & 486.0 & 19 & 0.20 & 0.98 & 486 & 486.0 & 18 & 0.11 & 2.45 \\
\hline & 9 & 170366 & 462 & 12 & 1749.66 & 2729.67 & 455 & 455.0 & 15 & 0.65 & 2.47 & 455 & 455.0 & 15 & 0.15 & 2.45 \\
\hline & 11 & 16301 & 456 & 10 & 88.87 & 140.18 & 444 & 444.0 & 14 & 0.49 & 0.95 & 444 & 444.0 & 13 & 0.09 & 2.26 \\
\hline \multirow{3}{*}{$\begin{array}{c}\text { KroA } \\
100\end{array}$} & 7 & $208101^{*}$ & 10306 & 15 & 6303.03 & 6475.95 & 9674 & 9674.0 & 19 & 0.52 & 1.06 & 9674 & 9674.0 & 19 & 0.36 & 2.78 \\
\hline & 9 & 95770 & 9573 & 12 & 524.49 & 1365.42 & 9159 & 9159.2 & 16 & 0.83 & 1.98 & 9159 & 9159.0 & 15 & 0.17 & 2.30 \\
\hline & 11 & 33444 & 9460 & 10 & 409.47 & 433.97 & 8901 & 8901.0 & 13 & 0.37 & 0.95 & 8901 & 8901.0 & 13 & 0.09 & 2.05 \\
\hline \multirow{3}{*}{ KroB 100} & 7 & 4068 & 11123 & 14 & 45.62 & 48.35 & 9537 & 9537.6 & 22 & 0.65 & 0.85 & 9537 & 9537.0 & 20 & 0.71 & 2.53 \\
\hline & 9 & 133396 & 9505 & 12 & 2112.57 & 2623.79 & 9240 & 9240.0 & 16 & 0.98 & 1.55 & 9240 & 9240.0 & 15 & 0.11 & 2.62 \\
\hline & 11 & $90000^{*}$ & 9049 & 10 & 1056.27 & 2895.35 & 8842 & 8842.0 & 15 & 0.26 & 0.88 & 8842 & 8842.0 & 13 & 0.10 & 2.48 \\
\hline \multirow{3}{*}{ KroC 100} & 7 & $129545^{*}$ & 10367 & 15 & 3391.82 & 4212.98 & 9723 & 9723.0 & 17 & 0.38 & 0.99 & 9723 & 9723.0 & 17 & 0.26 & 2.55 \\
\hline & 9 & 5028 & 9952 & 12 & 35.91 & 52.25 & 9171 & 9171.0 & 13 & 0.21 & 1.56 & 9171 & 9171.0 & 13 & 0.10 & 2.42 \\
\hline & 11 & 75987* & 9150 & 10 & 1389.84 & 2482.00 & 8632 & 8632.0 & 13 & 0.26 & 0.92 & 8632 & 8632.0 & 13 & 0.10 & 2.44 \\
\hline \multirow{3}{*}{$\begin{array}{c}\text { KroD } \\
100\end{array}$} & 7 & 1392 & 11085 & 14 & 10.29 & 15.58 & 9626 & 9629.0 & 20 & 0.56 & 0.96 & 9626 & 9626.0 & 20 & 0.19 & 2.36 \\
\hline & 9 & 700 & 10564 & 11 & 6.18 & 7.74 & 8885 & 8885.0 & 13 & 0.65 & 1.69 & 8885 & 8885.0 & 13 & 0.11 & 2.73 \\
\hline & 11 & $85147^{*}$ & 9175 & 10 & 968.39 & 2761.51 & 8725 & 8725.0 & 13 & 0.24 & 0.88 & 8725 & 8725.0 & 13 & 0.11 & 2.54 \\
\hline & 7 & 92414* & 11323 & 15 & 1971.32 & 3075.58 & 10150 & 10154.8 & 19 & 0.17 & 1.00 & 10150 & 10150.0 & 19 & 0.13 & 2.16 \\
\hline KroE 100 & 9 & $85305^{*}$ & 9095 & 12 & 1918.72 & 2764.70 & 8992 & 8995.2 & 14 & 0.26 & 1.57 & 8991 & 8991.0 & 14 & 0.11 & 2.39 \\
\hline & 11 & 70807* & 8936 & 10 & 609.81 & 2335.43 & 8450 & 8450.0 & 14 & 0.37 & 0.88 & 8450 & 8450.0 & 13 & 0.12 & 2.64 \\
\hline & 7 & 2520 & 4105 & 14 & 24.43 & 4196.23 & 3461 & 3463.0 & 18 & 0.16 & 1.00 & 3461 & 3485.6 & 18 & 0.66 & 2.30 \\
\hline Rd 100 & 9 & $95242 *$ & 3414 & 12 & 1798.14 & 3118.93 & 3194 & 3195.4 & 21 & 0.93 & 1.66 & 3194 & 3194.0 & 16 & 0.13 & 2.31 \\
\hline & 11 & 1291 & 3453 & 10 & 8.60 & 22.11 & 2922 & 2922.0 & 13 & 0.03 & 0.88 & 2922 & 2922.0 & 13 & 0.09 & 1.97 \\
\hline & 7 & 97785* & 12367 & 22 & 2252.50 & 3499.43 & 11426 & 11517.4 & 27 & 0.98 & 1.62 & 11423 & 11800.2 & 28 & 1.24 & 4.10 \\
\hline KroA150 & 9 & $69377^{*}$ & 11955 & 17 & 2454.99 & 2477.69 & 10072 & 10072.0 & 23 & 0.56 & 1.92 & 10056 & 10062.4 & 26 & 1.35 & 3.53 \\
\hline & 11 & $169846^{*}$ & 10564 & 15 & 5483.07 & 5518.26 & 9439 & 9440.6 & 21 & 0.32 & 1.20 & 9439 & 9439.0 & 21 & 0.61 & 3.46 \\
\hline & 7 & 14400 & 12876 & 21 & 196.85 & 270.94 & 11493 & 11577.6 & 30 & 1.31 & 1.60 & 11457 & 11491.2 & 30 & 1.55 & 4.30 \\
\hline KroB 150 & 9 & $137763^{*}$ & 11774 & 18 & 2760.03 & 4572.81 & 10121 & 10130.0 & 24 & 0.95 & 2.12 & 10121 & \begin{tabular}{|l|}
10121.0 \\
\end{tabular} & 24 & 0.40 & 3.50 \\
\hline & 11 & 1431 & 10968 & 14 & 26.64 & 46.96 & 9611 & 9648.6 & 22 & 0.81 & 1.23 & 9611 & 9611.0 & 21 & 0.15 & 3.69 \\
\hline & 7 & $53686^{*}$ & 14667 & 28 & 537.60 & 1170.37 & 13293 & 13327.5 & 34 & 1.42 & 2.67 & 13285 & 13666.4 & 35 & 0.43 & 5.64 \\
\hline $\begin{array}{l}\text { KroA } \\
200\end{array}$ & 9 & $64763 *$ & 12683 & 23 & 1504.07 & 1628.36 & 11726 & 11726.2 & 28 & 2.49 & 3.88 & 11708 & 11716.8 & 28 & 2.11 & 5.12 \\
\hline & 11 & $29668^{*}$ & 12736 & 19 & 398.25 & 671.55 & 10837 & 10837.0 & 28 & 1.15 & 1.78 & 10748 & 10848.6 & 29 & 1.43 & 4.61 \\
\hline & 7 & $107208^{*}$ & 14952 & 29 & 365.08 & 3351.89 & 13101 & 13197.6 & 36 & 1.52 & 2.67 & 13100 & 13511.6 & 36 & 0.10 & 5.42 \\
\hline KroB 200 & 9 & $38218^{*}$ & 13679 & 23 & 637.66 & 805.04 & 11900 & 11864.2 & 29 & 0.04 & 0.15 & 11900 & 11964.8 & 31 & 0.80 & 5.11 \\
\hline & 11 & $67896^{*}$ & 12265 & 20 & 493.64 & 1410.60 & 10676 & 10678.4 & 29 & 0.96 & 1.83 & 10676 & 10809.6 & 30 & 1.02 & 5.01 \\
\hline Avg & & 55896 & 9808.02 & 13 & 1017.94 & \begin{tabular}{|l|}
1626.68 \\
\end{tabular} & 9029.6 & 9037.5 & 17 & 0.51 & 1.34 & 9026.0 & 9060.5 & 17 & 0.35 & 2.56 \\
\hline No.Best & & & 1 & & & & 39 & & & & & 48 & & & & \\
\hline
\end{tabular}


Table 4. Comparison of LS1 and LS2 on Binary GCSP

\begin{tabular}{|c|c|c|c|c|c|c|c|c|c|c|c|}
\hline \multirow[b]{2}{*}{ Instance } & \multirow[b]{2}{*}{$\mathrm{NC}$} & \multicolumn{5}{|c|}{ LS1 } & \multicolumn{5}{|c|}{ LS2 } \\
\hline & & $\begin{array}{l}\text { Best } \\
\text { Cost }\end{array}$ & $\begin{array}{l}\text { Avg. } \\
\text { Cost }\end{array}$ & NB & $\begin{array}{c}\text { Avg. } \\
\text { TB }\end{array}$ & $\begin{array}{c}\text { Avg. } \\
\text { TT }\end{array}$ & $\begin{array}{l}\text { Best } \\
\text { Cost }\end{array}$ & $\begin{array}{l}\text { Avg. } \\
\text { Cost }\end{array}$ & NB & $\begin{array}{c}\text { Avg. } \\
\text { TB }\end{array}$ & $\begin{array}{c}\text { Avg. } \\
\text { TT }\end{array}$ \\
\hline \multirow{3}{*}{ Eil 51} & 7 & 1190 & 1210.4 & 19 & 0.32 & 1.28 & 1190 & 1190.0 & 19 & 0.29 & 1.59 \\
\hline & 9 & 991 & 991.8 & 15 & 0.97 & 1.58 & 991 & 999.6 & 15 & 0.35 & 1.57 \\
\hline & 11 & 844 & 845.2 & 13 & 0.23 & 1.04 & 844 & 853.6 & 13 & 0.20 & 1.55 \\
\hline \multirow{3}{*}{ Berlin 52} & 7 & 5429 & 5429.0 & 17 & 0.37 & 1.24 & 5429 & 5619.8 & 17 & 0.26 & 1.57 \\
\hline & 9 & 4807 & 4807.0 & 14 & 0.19 & 1.04 & 4807 & 4807.0 & 14 & 0.06 & 1.53 \\
\hline & 11 & 4590 & 4590.0 & 13 & 0.24 & 1.05 & 4590 & 4590.0 & 13 & 0.09 & 1.31 \\
\hline \multirow{3}{*}{ St 70} & 7 & 1831 & 1831.0 & 29 & 0.78 & 2.13 & 1837 & 1837.6 & 29 & 0.58 & 1.97 \\
\hline & 9 & 1461 & 1461.0 & 22 & 0.17 & 1.59 & 1460 & 1466.4 & 22 & 0.19 & 2.05 \\
\hline & 11 & 1268 & 1268.0 & 19 & 0.71 & 1.35 & 1268 & 1273.2 & 19 & 0.14 & 2.00 \\
\hline \multirow{3}{*}{ Eil 76} & 7 & 1610 & 1612.6 & 26 & 0.95 & 1.91 & 1651 & 1652.6 & 27 & 0.63 & 2.25 \\
\hline & 9 & 1278 & 1294.6 & 20 & 0.90 & 1.58 & 1297 & 1302.8 & 21 & 1.05 & 2.46 \\
\hline & 11 & 1117 & 1117.0 & 18 & 0.12 & 2.21 & 1117 & 1122.6 & 18 & 0.56 & 2.13 \\
\hline \multirow{3}{*}{$\operatorname{Pr} 76$} & 7 & 66455 & 66616.6 & 29 & 1.19 & 1.79 & 66455 & 66455.0 & 29 & 0.29 & 2.39 \\
\hline & 9 & 62494 & 62524.0 & 25 & 0.87 & 1.59 & 62907 & 63155.8 & 25 & 0.27 & 2.36 \\
\hline & 11 & 52175 & 52175.0 & 19 & 0.59 & 1.33 & 52175 & 52303.6 & 19 & 0.19 & 1.97 \\
\hline \multirow{3}{*}{ Rat 99} & 7 & 2341 & 2345.8 & 34 & 1.93 & 2.51 & 2341 & 2345.2 & 34 & 0.99 & 4.36 \\
\hline & 9 & 1938 & 1942.6 & 27 & 1.36 & 2.21 & 1934 & 1950.4 & 27 & 1.08 & 3.50 \\
\hline & 11 & 1712 & 1713.2 & 24 & 0.82 & 2.64 & 1683 & 1709.2 & 23 & 0.69 & 3.52 \\
\hline \multirow{3}{*}{$\begin{array}{c}\text { KroA } \\
100\end{array}$} & 7 & 14660 & 14660.0 & 41 & 1.68 & 2.89 & 14660 & 14790.4 & 41 & 0.87 & 5.25 \\
\hline & 9 & 12974 & 12974.0 & 33 & 0.49 & 2.00 & 12974 & 12974.0 & 33 & 0.32 & 0.16 \\
\hline & 11 & 11942 & 11950.8 & 29 & 1.91 & 2.32 & 11942 & 12118.6 & 29 & 0.39 & 3.46 \\
\hline \multirow{3}{*}{$\begin{array}{c}\text { KroB } \\
100\end{array}$} & 7 & 14390 & 14469.8 & 42 & 1.65 & 2.83 & 14323 & 14511.0 & 44 & 1.84 & 5.32 \\
\hline & 9 & 12223 & 12223.0 & 34 & 0.62 & 2.11 & 12222 & 12225.0 & 33 & 0.73 & 3.43 \\
\hline & 11 & 11276 & 11277.2 & 28 & 0.92 & 2.10 & 11276 & 11276.0 & 28 & 1.04 & 3.50 \\
\hline \multirow{3}{*}{$\begin{array}{c}\text { KroC } \\
100\end{array}$} & 7 & 13830 & 13831.4 & 41 & 0.78 & 2.85 & 13830 & 13830.0 & 41 & 0.57 & 5.30 \\
\hline & 9 & 12149 & 12154.0 & 33 & 0.76 & 1.85 & 12149 & 12197.2 & 33 & 0.45 & 3.26 \\
\hline & 11 & 11032 & 11032.0 & 26 & 0.10 & 2.02 & 11032 & 11032.0 & 26 & 0.39 & 3.09 \\
\hline \multirow{3}{*}{$\begin{array}{c}\text { KroD } \\
100\end{array}$} & 7 & 13567 & 13584.8 & 38 & 1.92 & 2.75 & 13567 & 13917.0 & 38 & 1.52 & 5.31 \\
\hline & 9 & 12413 & 12414.0 & 32 & 0.84 & 2.24 & 12409 & 12437.4 & 31 & 0.65 & 3.62 \\
\hline & 11 & 11486 & 11491.0 & 28 & 1.50 & 1.90 & 11443 & 11483.8 & 29 & 0.33 & 3.63 \\
\hline \multirow{3}{*}{$\begin{array}{c}\text { KroE } \\
100\end{array}$} & 7 & 15321 & 15333.0 & 41 & 1.96 & 3.04 & 15357 & 15426.6 & 40 & 0.50 & 3.20 \\
\hline & 9 & 12482 & 12482.0 & 32 & 0.84 & 4.64 & 12482 & 12482.0 & 32 & 0.36 & 3.33 \\
\hline & 11 & 11429 & 11461.4 & 29 & 0.63 & 1.81 & 11487 & 11487.0 & 29 & 0.85 & 3.69 \\
\hline \multirow{3}{*}{$\mathrm{Rd} 100$} & 7 & 6174 & 6188.6 & 37 & 2.08 & 2.77 & 6209 & 6243.0 & 38 & 0.78 & 3.47 \\
\hline & 9 & 5469 & 5498.2 & 29 & 1.55 & 5.03 & 5454 & 5596.8 & 29 & 0.58 & 3.42 \\
\hline & 11 & 4910 & 4940.6 & 28 & 0.77 & 1.84 & 4910 & 4914.8 & 28 & 0.58 & 3.29 \\
\hline \multirow{3}{*}{ KroA150 } & 7 & 17214 & 17221.0 & 56 & 2.68 & 6.15 & 17258 & 17338.6 & 55 & 1.98 & 5.62 \\
\hline & 9 & 15030 & 15067.4 & 46 & 3.32 & 9.95 & 15007 & 15280.6 & 46 & 1.49 & 6.09 \\
\hline & 11 & 13666 & 13666.0 & 40 & 2.85 & 3.67 & 13743 & 13987.8 & 40 & 1.68 & 5.48 \\
\hline & 7 & 17712 & 17778.8 & 59 & 6.05 & 6.44 & 17700 & 18017.8 & 60 & 2.59 & 5.47 \\
\hline $\begin{array}{c}\text { KroB } \\
150\end{array}$ & 9 & 15472 & 15532.0 & 50 & 3.63 & 9.55 & 15505 & 15940.2 & 50 & 1.29 & 5.85 \\
\hline & 11 & 13719 & 13727.2 & 44 & 2.41 & 4.81 & 13738 & 13825.0 & 41 & 1.72 & 6.02 \\
\hline & 7 & 21410 & 21476.0 & 78 & 8.95 & 13.88 & 21433 & 21493.6 & 79 & 2.87 & 7.60 \\
\hline KroA & 9 & 17912 & 18004.2 & 57 & 7.44 & 0.26 & 18131 & 18292.4 & 60 & 1.92 & 7.69 \\
\hline & 11 & 16478 & 16565.6 & 58 & 6.68 & 8.73 & 16556 & 16729.6 & 55 & 2.77 & 7.18 \\
\hline & 7 & 20864 & 20973.6 & 79 & 7.01 & 15.89 & 20769 & 20973.0 & 79 & 1.64 & 7.80 \\
\hline KroB & 9 & 18284 & 18361.0 & 66 & 7.10 & 0.27 & 18249 & 18580.6 & 67 & 1.65 & 7.75 \\
\hline & 11 & 16078 & 16108.0 & 55 & 6.83 & 7.29 & 16024 & 16152.0 & 55 & 4.42 & 6.84 \\
\hline Avg & & 13022.9 & 13046.3 & 35 & 2.06 & 3.42 & 13037.8 & 13128.9 & 35 & 0.97 & 3.86 \\
\hline No.Best & & 35 & & & & & 34 & & & & \\
\hline
\end{tabular}


Table 5. Comparison of LS1 and LS2 on Integer GCSP without overnights

\begin{tabular}{|c|c|c|c|c|c|c|c|c|c|c|c|c|c|}
\hline \multirow[b]{2}{*}{ Instance } & \multirow[b]{2}{*}{$\mathrm{NC}$} & \multicolumn{6}{|c|}{ LS1 } & \multicolumn{6}{|c|}{ LS2 } \\
\hline & & $\begin{array}{l}\text { Best } \\
\text { Cost }\end{array}$ & $\begin{array}{l}\text { Avg. } \\
\text { Cost }\end{array}$ & NB & NR & $\begin{array}{c}\text { Avg. } \\
\text { TB }\end{array}$ & $\begin{array}{l}\text { Avg. } \\
\text { TT }\end{array}$ & $\begin{array}{l}\text { Best } \\
\text { Cost }\end{array}$ & $\begin{array}{l}\text { Avg. } \\
\text { Cost }\end{array}$ & NB & NR & $\begin{array}{c}\text { Avg. } \\
\text { TB }\end{array}$ & $\begin{array}{c}\text { Avg. } \\
\text { TT }\end{array}$ \\
\hline \multirow{3}{*}{ Eil 51} & 7 & 1185 & 1185.0 & 19 & 1 & 0.87 & 3.07 & 1185 & 1187.0 & 19 & 1 & 1.46 & 5.43 \\
\hline & 9 & 991 & 991.2 & 15 & 0 & 0.86 & 2.56 & 991 & 994.8 & 15 & 0 & 1.54 & 4.64 \\
\hline & 11 & 843 & 843.8 & 13 & 1 & 0.14 & 2.27 & 843 & 843.2 & 13 & 1 & 0.96 & 4.49 \\
\hline \multirow{3}{*}{ Berlin 52} & 7 & 5429 & 5429.0 & 17 & 0 & 0.17 & 2.65 & 5429 & 5603.6 & 17 & 0 & 0.76 & 4.64 \\
\hline & 9 & 4785 & 4785.0 & 15 & 1 & 0.45 & 2.70 & 4785 & 4807.6 & 15 & 1 & 0.09 & 3.99 \\
\hline & 11 & 4590 & 4599.6 & 13 & 0 & 0.90 & 2.26 & 4590 & 4599.6 & 13 & 0 & 0.10 & 3.51 \\
\hline \multirow{3}{*}{ St 70} & 7 & 1778 & 1778.0 & 28 & 3 & 3.36 & 5.05 & 1778 & 1779.6 & 28 & 3 & 0.34 & 7.13 \\
\hline & 9 & 1461 & 1461.0 & 22 & 0 & 1.25 & 4.17 & 1460 & 1464.2 & 22 & 0 & 0.95 & 6.14 \\
\hline & 11 & 1241 & 1251.8 & 18 & 1 & 0.55 & 3.27 & 1241 & 1253.8 & 18 & 1 & 1.16 & 5.58 \\
\hline \multirow{3}{*}{ Eil 76} & 7 & 1600 & 1600.6 & 26 & 1 & 3.46 & 4.73 & 1600 & 1627.4 & 26 & 1 & 1.72 & 6.49 \\
\hline & 9 & 1268 & 1274.0 & 20 & 0 & 2.18 & 3.38 & 1268 & 1285.8 & 20 & 0 & 2.80 & 6.80 \\
\hline & 11 & 1117 & 1117.0 & 18 & 0 & 0.06 & 3.65 & 1117 & 1128.2 & 18 & 0 & 0.66 & 6.39 \\
\hline \multirow{3}{*}{$\operatorname{Pr} 76$} & 7 & 65721 & 65902.4 & 28 & 1 & 1.61 & 7.07 & 64111 & 64286.0 & 29 & 4 & 0.39 & 7.70 \\
\hline & 9 & 55129 & 57078.0 & 26 & 4 & 1.36 & 3.91 & 54859 & 55137.6 & 27 & 5 & 0.45 & 6.33 \\
\hline & 11 & 49559 & 50478.2 & 21 & 1 & 1.25 & 3.09 & 49445 & 49589.0 & 21 & 3 & 1.82 & 5.62 \\
\hline \multirow{3}{*}{ Rat 99} & 7 & 2311 & 2314.4 & 33 & 1 & 4.80 & 6.24 & 2311 & 2335.8 & 33 & 1 & 2.41 & 10.10 \\
\hline & 9 & 1937 & 1939.4 & 27 & 0 & 4.43 & 7.53 & 1936 & 1951.8 & 27 & 0 & 0.48 & 9.25 \\
\hline & 11 & 1683 & 1700.4 & 23 & 0 & 2.02 & 3.73 & 1686 & 1704.0 & 23 & 0 & 0.40 & 9.28 \\
\hline \multirow{3}{*}{$\begin{array}{c}\text { KroA } \\
100\end{array}$} & 7 & 14660 & 14660.0 & 41 & 0 & 1.68 & 10.20 & 14660 & 14721.8 & 41 & 0 & 0.60 & 11.73 \\
\hline & 9 & 12974 & 12974.0 & 33 & 0 & 0.30 & 5.75 & 12974 & 12974.0 & 33 & 0 & 2.14 & 10.22 \\
\hline & 11 & 11737 & 11737.0 & 31 & 1 & 2.44 & 5.87 & 11737 & 11737.0 & 29 & 1 & 0.69 & 9.51 \\
\hline \multirow{3}{*}{$\begin{array}{c}\text { KroB } \\
100\end{array}$} & 7 & 14203 & 14284.8 & 43 & 3 & 4.92 & 7.41 & 14209 & 14288.8 & 43 & 2 & 2.79 & 12.77 \\
\hline & 9 & 12172 & 12200.4 & 35 & 2 & 1.43 & 4.57 & 12200 & 12200.0 & 34 & 3 & 3.68 & 10.36 \\
\hline & 11 & 11268 & 11268.0 & 27 & 2 & 1.99 & 4.63 & 11268 & 11468.4 & 27 & 2 & 4.07 & 9.90 \\
\hline \multirow{3}{*}{$\begin{array}{c}\text { KroC } \\
100\end{array}$} & 7 & 13525 & 13671.4 & 42 & 5 & 3.74 & 6.53 & 13830 & 13938.0 & 41 & 0 & 0.76 & 12.53 \\
\hline & 9 & 12119 & 12119.0 & 33 & 1 & 1.92 & 4.85 & 12165 & 12165.0 & 33 & 1 & 0.59 & 10.71 \\
\hline & 11 & 11032 & 11032.0 & 26 & 0 & 1.01 & 5.46 & 11032 & 11042.6 & 26 & 0 & 1.12 & 8.53 \\
\hline \multirow{3}{*}{$\begin{array}{c}\text { KroD } \\
100\end{array}$} & 7 & 13501 & 13501.0 & 39 & 2 & 1.70 & 6.51 & 13501 & 13621.8 & 39 & 2 & 2.36 & 13.06 \\
\hline & 9 & 12257 & 12260.2 & 31 & 1 & 2.19 & 5.26 & 12261 & 12279.2 & 31 & 1 & 0.76 & 10.97 \\
\hline & 11 & 11450 & 11467.0 & 30 & 1 & 2.59 & 4.69 & 11409 & 11434.4 & 30 & 1 & 2.42 & 9.98 \\
\hline \multirow{3}{*}{$\begin{array}{c}\text { KroE } \\
100\end{array}$} & 7 & 15308 & 15327.4 & 43 & 1 & 3.38 & 6.68 & 15357 & 15633.0 & 40 & 0 & 3.12 & 11.44 \\
\hline & 9 & 12482 & 12482.2 & 32 & 0 & 0.37 & 5.21 & 12482 & 12484.0 & 32 & 0 & 0.63 & 9.97 \\
\hline & 11 & 11367 & 11401.2 & 28 & 1 & 2.27 & 4.14 & 11344 & 11462.0 & 30 & 1 & 1.89 & 9.41 \\
\hline \multirow{3}{*}{$\mathrm{Rd} 100$} & 7 & 6078 & 6104.2 & 37 & 2 & 3.55 & 6.91 & 6147 & 6256.4 & 36 & 1 & 1.09 & 10.87 \\
\hline & 9 & 5384 & 5391.0 & 31 & 2 & 3.62 & 7.70 & 5384 & 5443.0 & 30 & 2 & 4.54 & 10.15 \\
\hline & 11 & 4853 & 4864.8 & 29 & 2 & 2.74 & 4.07 & 4853 & 4901.6 & 29 & 1 & 0.33 & 8.74 \\
\hline & 7 & 16947 & 16959.0 & 57 & 4 & 5.71 & 14.94 & 16947 & 17226.8 & 57 & 4 & 8.54 & 18.99 \\
\hline KroA 150 & 9 & 15007 & 15025.8 & 46 & 0 & 5.61 & 11.25 & 15007 & 15080.8 & 46 & 0 & 2.96 & 18.09 \\
\hline & 11 & 13580 & 13608.8 & 40 & 2 & 3.77 & 8.75 & 13580 & 13811.0 & 40 & 2 & 3.88 & 14.99 \\
\hline & 7 & 17620 & 17714.8 & 58 & 1 & 9.08 & 14.94 & 17621 & 17832.0 & 59 & 1 & 4.36 & 16.85 \\
\hline $\begin{array}{c}\text { KroB } \\
150\end{array}$ & 9 & 15332 & 15332.0 & 49 & 3 & 7.47 & 11.51 & 15332 & 15493.4 & 48 & 3 & 6.90 & 16.71 \\
\hline & 11 & 13554 & 13596.8 & 41 & 2 & 4.30 & 9.04 & 13554 & 13623.6 & 41 & 2 & 3.48 & 15.38 \\
\hline & 7 & 20979 & 21168.8 & 77 & 3 & 16.19 & 30.16 & 21115 & 21429.6 & 78 & 4 & 4.17 & 24.25 \\
\hline KroA & 9 & 17657 & 17901.4 & 62 & 2 & 12.22 & 21.73 & 17867 & 18291.4 & 63 & 4 & 9.68 & 22.23 \\
\hline & 11 & 16280 & 16298.0 & 57 & 3 & 14.08 & 17.98 & 16453 & 16663.4 & 52 & 2 & 3.49 & 20.15 \\
\hline & 7 & 20636 & 20719.2 & 78 & 3 & 18.88 & 31.28 & 20817 & 21198.2 & 81 & 3 & 8.01 & 24.12 \\
\hline $\begin{array}{c}\text { KroB } \\
200\end{array}$ & 9 & 18230 & 18324.6 & 66 & 1 & 17.42 & 23.12 & 18124 & 18251.4 & 66 & 6 & 7.86 & 22.22 \\
\hline & 11 & 15725 & 15860.6 & 55 & 3 & 11.14 & 17.32 & 15801 & 16115.0 & 57 & 5 & 4.67 & 19.74 \\
\hline Avg & & 12719.7 & 12812.2 & 35 & 1 & 4.11 & 8.12 & 12701.4 & 12805.1 & 35 & 2 & 2.50 & 11.21 \\
\hline No.Best & & 40 & & & & & & 33 & & & & & \\
\hline
\end{tabular}


Table 6. Comparison of LS1 and LS2 on Integer GCSP with overnights

\begin{tabular}{|c|c|c|c|c|c|c|c|c|c|c|c|c|c|}
\hline \multirow[b]{2}{*}{ Instance } & \multirow[b]{2}{*}{$\mathrm{NC}$} & \multicolumn{6}{|c|}{ LS1 } & \multicolumn{6}{|c|}{ LS2 } \\
\hline & & $\begin{array}{l}\text { Best } \\
\text { Cost }\end{array}$ & $\begin{array}{l}\text { Avg. } \\
\text { Cost }\end{array}$ & NB & $\mathrm{ON}$ & $\begin{array}{c}\text { Avg. } \\
\text { TB }\end{array}$ & $\begin{array}{c}\text { Avg. } \\
\text { TT }\end{array}$ & $\begin{array}{l}\text { Best } \\
\text { Cost }\end{array}$ & $\begin{array}{l}\text { Avg. } \\
\text { Cost }\end{array}$ & NB & ON & $\begin{array}{c}\text { Avg. } \\
\text { TB }\end{array}$ & $\begin{array}{c}\text { Avg. } \\
\text { TT }\end{array}$ \\
\hline \multirow{3}{*}{ Eil 51} & 7 & 1146 & 1846.0 & 19 & 10 & 0.39 & 6.84 & 1146 & 1146.0 & 19 & 10 & 0.53 & 4.05 \\
\hline & 9 & 958 & 964.4 & 15 & 5 & 1.25 & 6.16 & 958 & 973.4 & 15 & 5 & 1.39 & 4.10 \\
\hline & 11 & 827 & 827.0 & 13 & 4 & 2.08 & 5.44 & 827 & 835.0 & 13 & 4 & 1.41 & 3.86 \\
\hline \multirow{3}{*}{ Berlin 52} & 7 & 4966 & 4966.0 & 18 & 9 & 0.15 & 4.65 & 4966 & 4972.2 & 18 & 9 & 0.31 & 3.92 \\
\hline & 9 & 4272 & 4274.2 & 17 & 8 & 2.09 & 4.79 & 4272 & 4295.4 & 16 & 7 & 0.28 & 3.41 \\
\hline & 11 & 3962 & 3962.0 & 14 & 8 & 2.03 & 5.71 & 3962 & 3962.0 & 14 & 8 & 0.31 & 3.11 \\
\hline \multirow{3}{*}{ St 70} & 7 & 1654 & 1654.2 & 27 & 15 & 5.14 & 10.46 & 1654 & 1656.2 & 27 & 14 & 1.08 & 5.89 \\
\hline & 9 & 1417 & 1437.0 & 22 & 10 & 0.70 & 9.48 & 1416 & 1439.2 & 22 & 9 & 1.74 & 5.37 \\
\hline & 11 & 1196 & 1200.2 & 18 & 6 & 3.93 & 7.94 & 1196 & 1197.4 & 18 & 6 & 0.86 & 5.14 \\
\hline \multirow{3}{*}{ Eil 76} & 7 & 1554 & 1558.8 & 26 & 10 & 4.72 & 14.77 & 1561 & 1599.2 & 26 & 5 & 1.07 & 6.47 \\
\hline & 9 & 1284 & 1296.4 & 21 & 7 & 6.27 & 11.83 & 1284 & 1314.0 & 21 & 4 & 1.02 & 5.71 \\
\hline & 11 & 1108 & 1111.6 & 18 & 5 & 7.37 & 11.39 & 1107 & 1109.0 & 18 & 3 & 1.10 & 5.77 \\
\hline \multirow{3}{*}{$\operatorname{Pr} 76$} & 7 & 52427 & 53163.6 & 32 & 17 & 4.80 & 10.92 & 52265 & 53153.8 & 31 & 17 & 1.19 & 6.16 \\
\hline & 9 & 47092 & 47764.0 & 29 & 17 & 3.02 & 7.11 & 47234 & 47609.4 & 28 & 16 & 1.56 & 5.60 \\
\hline & 11 & 44028 & 44028.0 & 20 & 10 & 1.63 & 6.00 & 44028 & 44028.0 & 20 & 10 & 1.34 & 4.57 \\
\hline \multirow{3}{*}{ Rat 99} & 7 & 2233 & 2242.0 & 32 & 11 & 7.53 & 11.14 & 2218 & 2242.8 & 32 & 8 & 6.14 & 9.58 \\
\hline & 9 & 1895 & 1901.6 & 27 & 8 & 7.98 & 11.64 & 1922 & 1942.8 & 28 & 8 & 2.62 & 8.59 \\
\hline & 11 & 1673 & 1676.8 & 24 & 9 & 7.80 & 10.19 & 1650 & 1678.6 & 23 & 5 & 2.21 & 8.22 \\
\hline \multirow{3}{*}{$\begin{array}{c}\text { KroA } \\
100\end{array}$} & 7 & 12006 & 12182.8 & 43 & 26 & 10.38 & 18.91 & 12006 & 12096.6 & 43 & 26 & 1.20 & 8.32 \\
\hline & 9 & 11653 & 11733.4 & 33 & 18 & 10.33 & 17.68 & 11185 & 11259.4 & 36 & 23 & 0.83 & 7.84 \\
\hline & 11 & 10665 & 10688.0 & 31 & 18 & 9.81 & 16.83 & 10701 & 10739.2 & 30 & 18 & 0.41 & 7.27 \\
\hline \multirow{3}{*}{$\begin{array}{c}\text { KroB } \\
100\end{array}$} & 7 & 12373 & 12605.4 & 41 & 23 & 8.48 & 12.72 & 12363 & 12665.6 & 45 & 28 & 0.89 & 9.43 \\
\hline & 9 & 11157 & 11200.2 & 35 & 20 & 5.01 & 11.65 & 11128 & 11320.0 & 35 & 21 & 2.17 & 9.12 \\
\hline & 11 & 10302 & 10302.0 & 29 & 16 & 4.06 & 10.32 & 10302 & 10380.6 & 28 & 14 & 3.76 & 8.28 \\
\hline \multirow{3}{*}{$\begin{array}{c}\text { KroC } \\
100\end{array}$} & 7 & 12223 & 12224.4 & 41 & 22 & 9.09 & 15.87 & 11996 & 12060.8 & 45 & 28 & 1.50 & 8.55 \\
\hline & 9 & 11116 & 11160.8 & 33 & 17 & 7.54 & 15.09 & 11084 & 11142.2 & 35 & 20 & 2.06 & 7.69 \\
\hline & 11 & 10299 & 10330.2 & 28 & 15 & 3.29 & 11.61 & 10421 & 10463.0 & 29 & 17 & 0.36 & 6.72 \\
\hline \multirow{3}{*}{$\begin{array}{c}\text { KroD } \\
100\end{array}$} & 7 & 11725 & 11857.6 & 39 & 21 & 4.92 & 12.99 & 11725 & 11849.2 & 39 & 21 & 2.37 & 9.06 \\
\hline & 9 & 11039 & 11108.8 & 30 & 17 & 7.09 & 13.19 & 10787 & 10787.0 & 34 & 21 & 2.45 & 7.80 \\
\hline & 11 & 10352 & 10405.2 & 30 & 18 & 8.57 & 12.18 & 10426 & 10447.6 & 30 & 17 & 0.60 & 7.57 \\
\hline \multirow{3}{*}{$\begin{array}{c}\text { KroE } \\
100\end{array}$} & 7 & 13273 & 13273.0 & 43 & 26 & 7.49 & 15.36 & 12438 & 12696.4 & 45 & 26 & 4.80 & 8.33 \\
\hline & 9 & 10821 & 10926.2 & 34 & 20 & 8.08 & 14.79 & 10821 & 10906.2 & 34 & 20 & 1.53 & 7.13 \\
\hline & 11 & 10010 & 10065.8 & 30 & 17 & 6.32 & 9.27 & 10007 & 10094.2 & 29 & 17 & 1.55 & 6.85 \\
\hline \multirow{3}{*}{$\mathrm{Rd} 100$} & 7 & 5634 & 5654.4 & 37 & 17 & 12.53 & 14.43 & 5567 & 5681.4 & 39 & 20 & 3.32 & 8.63 \\
\hline & 9 & 4950 & 5034.4 & 32 & 18 & 9.68 & 9.78 & 4974 & 5059.4 & 32 & 16 & 0.71 & 7.79 \\
\hline & 11 & 4514 & 4519.8 & 27 & 12 & 7.31 & 9.48 & 4462 & 4563.8 & 29 & 16 & 2.91 & 7.15 \\
\hline & 7 & 15335 & 15381.4 & 57 & 28 & 19.20 & 25.73 & 14936 & 15293.2 & 60 & 34 & 4.47 & 14.61 \\
\hline KroA 150 & 9 & 13484 & 13533.4 & 50 & 27 & 17.08 & 22.29 & 13115 & 13386.0 & 52 & 30 & 5.21 & 14.17 \\
\hline & 11 & 12112 & 12300.4 & 41 & 18 & 10.51 & 17.64 & 12211 & 12384.2 & 44 & 22 & 2.91 & 11.89 \\
\hline & 7 & 15242 & 15747.4 & 59 & 29 & 21.14 & 27.39 & 15162 & 15353.8 & 62 & 33 & 6.49 & 14.79 \\
\hline $\begin{array}{c}\text { KroB } \\
150\end{array}$ & 9 & 13242 & 13313.8 & 50 & 38 & 14.18 & 24.48 & 13198 & 13519.4 & 52 & 30 & 3.07 & 12.71 \\
\hline & 11 & 12418 & 12418.0 & 43 & 24 & 15.26 & 20.85 & 12183 & 12379.4 & 46 & 25 & 5.21 & 11.95 \\
\hline & 7 & 17839 & 17994.0 & 77 & 37 & 40.08 & 54.89 & 17683 & 18222.0 & 80 & 44 & 8.23 & 19.80 \\
\hline $\begin{array}{c}\text { KroA } \\
200\end{array}$ & 9 & 15563 & 15726.6 & 64 & 34 & 26.26 & 40.67 & 15523 & 15760.6 & 65 & 36 & 6.99 & 17.32 \\
\hline & 11 & 14430 & 14667.8 & 57 & 34 & 20.14 & 33.16 & 14302 & 14518.4 & 59 & 37 & 6.09 & 15.02 \\
\hline & 7 & 18421 & 18488.8 & 80 & 47 & 55.55 & 68.05 & 17873 & 18117.6 & 84 & 49 & 4.98 & 19.71 \\
\hline $\begin{array}{c}\text { KroB } \\
200\end{array}$ & 9 & 15935 & 16176.8 & 64 & 36 & 32.55 & 42.62 & 16069 & 16164.2 & 69 & 37 & 5.11 & 17.49 \\
\hline & 11 & 14235 & 14323.6 & 54 & 28 & 22.79 & 31.49 & 14064 & 14455.2 & 58 & 32 & 6.79 & 15.72 \\
\hline Avg & & 11167.92 & 11275.38 & 36 & 19 & 10.49 & 16.83 & 11091.21 & 11227.52 & 37 & 19 & 2.61 & 8.92 \\
\hline No.Best & & 22 & & & & & & 39 & & & & & \\
\hline
\end{tabular}


Table 7. Comparison of LS1 and LS2 on Steiner CSP

\begin{tabular}{|c|c|c|c|c|c|c|c|c|c|c|}
\hline \multirow{2}{*}{ Instance } & \multicolumn{5}{|c|}{ LS1 } & \multicolumn{5}{|c|}{ LS2 } \\
\hline & Best Cost & Avg. Cost & $\mathrm{NB}$ & Avg. TB & Avg. TT & Best Cost & Avg. Cost & NB & Avg. TB & Avg. TT \\
\hline \multirow{3}{*}{ Eil 51} & 163 & 163.0 & 8 & 0.03 & 0.79 & 163 & 163.0 & 8 & 0.05 & 1.04 \\
\hline & 159 & 159.0 & 8 & 0.08 & 0.79 & 159 & 159.0 & 9 & 0.04 & 0.75 \\
\hline & 147 & 147.0 & 7 & 0.06 & 0.79 & 147 & 147.0 & 7 & 0.04 & 0.87 \\
\hline \multirow{3}{*}{ Berlin 52} & 3470 & 3883.2 & 10 & 0.46 & 0.76 & 3470 & 3470.0 & 10 & 0.13 & 0.97 \\
\hline & 3097 & 3097.4 & 7 & 0.27 & 0.89 & 3097 & 3097.0 & 7 & 0.04 & 0.80 \\
\hline & 2956 & 2956.0 & 6 & 0.11 & 1.01 & 2956 & 2956.0 & 6 & 0.04 & 0.79 \\
\hline \multirow{3}{*}{ St 70} & 287 & 287.4 & 12 & 0.14 & 0.96 & 287 & 287.0 & 12 & 0.07 & 1.31 \\
\hline & 259 & 259.0 & 10 & 0.01 & 0.89 & 259 & 259.0 & 9 & 0.06 & 1.60 \\
\hline & 245 & 245.0 & 12 & 0.70 & 1.56 & 245 & 245.0 & 10 & 0.05 & 1.23 \\
\hline \multirow{3}{*}{ Eil 76} & 207 & 207.0 & 15 & 0.06 & 0.95 & 207 & 207.0 & 15 & 0.07 & 1.32 \\
\hline & 185 & 185.4 & 10 & 0.35 & 1.06 & 185 & 185.0 & 12 & 0.06 & 1.51 \\
\hline & 169 & 169.0 & 11 & 0.13 & 0.78 & 169 & 169.0 & 9 & 0.06 & 1.43 \\
\hline \multirow{3}{*}{$\operatorname{Pr} 76$} & 49773 & 49773.0 & 13 & 0.10 & 0.97 & 49773 & 49773.0 & 13 & 0.15 & 1.86 \\
\hline & 44889 & 44889.0 & 12 & 0.11 & 1.04 & 44889 & 44889.0 & 12 & 0.09 & 1.65 \\
\hline & 42950 & 42998.8 & 9 & 0.30 & 0.85 & 42950 & 42950.0 & 9 & 0.06 & 1.34 \\
\hline \multirow{3}{*}{ Rat 99} & 482 & 482.0 & 18 & 0.33 & 1.23 & 482 & 482.0 & 18 & 0.43 & 2.63 \\
\hline & 454 & 454.0 & 14 & 0.34 & 1.18 & 454 & 454.0 & 14 & 0.15 & 2.63 \\
\hline & 444 & 444.6 & 12 & 0.37 & 1.11 & 444 & 444.0 & 12 & 0.09 & 2.18 \\
\hline \multirow{3}{*}{ KroA 100} & 9545 & 9545.0 & 19 & 0.37 & 1.30 & 9545 & 9545.0 & 18 & 0.52 & 2.71 \\
\hline & 9112 & 9112.0 & 16 & 0.44 & 1.03 & 9112 & 9112.0 & 15 & 0.12 & 2.30 \\
\hline & 8833 & 8833.0 & 13 & 0.10 & 1.11 & 8833 & 8833.0 & 13 & 0.09 & 2.01 \\
\hline \multirow{3}{*}{ KroB 100} & 9536 & 9536.0 & 21 & 0.79 & 1.21 & 9536 & 9536.0 & 19 & 0.48 & 2.66 \\
\hline & 9199 & 9230.6 & 17 & 0.51 & 0.95 & 9199 & 9199.0 & 15 & 0.10 & 2.49 \\
\hline & 8763 & 8763.0 & 11 & 0.11 & 1.14 & 8763 & 8763.0 & 11 & 0.10 & 2.29 \\
\hline \multirow{3}{*}{ KroC 100} & 9591 & 9591.0 & 15 & 0.12 & 1.07 & 9590 & 9590.0 & 16 & 0.18 & 2.68 \\
\hline & 9171 & 9171.0 & 13 & 0.34 & 1.05 & 9171 & 9171.0 & 13 & 0.19 & 2.35 \\
\hline & 8632 & 8632.0 & 13 & 0.27 & 1.16 & 8632 & 8632.0 & 13 & 0.12 & 2.48 \\
\hline \multirow{3}{*}{ KroD 100} & 9526 & 9526.0 & 19 & 0.31 & 1.35 & 9526 & 9526.0 & 19 & 0.28 & 2.33 \\
\hline & 8885 & 8885.0 & 16 & 0.30 & 0.98 & 8885 & 8885.0 & 13 & 0.12 & 2.76 \\
\hline & 8725 & 8725.0 & 13 & 0.26 & 1.12 & 8725 & 8725.0 & 13 & 0.11 & 2.44 \\
\hline \multirow{3}{*}{ KroE 100} & 9800 & 9800.0 & 16 & 0.11 & 1.15 & 9800 & 9800.0 & 16 & 0.12 & 2.32 \\
\hline & 8987 & 8987.0 & 13 & 0.09 & 0.98 & 8986 & 8986.0 & 14 & 0.10 & 2.35 \\
\hline & 8450 & 8450.0 & 13 & 0.10 & 1.23 & 8450 & 8450.0 & 13 & 0.11 & 2.54 \\
\hline \multirow{3}{*}{ Rd 100} & 3412 & 3412.4 & 18 & 0.28 & 1.25 & 3412 & 3445.6 & 18 & 0.15 & 2.31 \\
\hline & 3194 & 3194.0 & 17 & 0.32 & 0.93 & 3194 & 3194.0 & 16 & 0.32 & 2.24 \\
\hline & 2761 & 2761.0 & 12 & 0.04 & 1.20 & 2761 & 2761.0 & 12 & 0.08 & 1.91 \\
\hline \multirow{3}{*}{ KroA150 } & 10939 & 10939.6 & 28 & 0.68 & 1.84 & 10939 & 10999.0 & 27 & 1.00 & 3.86 \\
\hline & 9808 & 9809.4 & 23 & 0.49 & 1.29 & 9808 & 9808.0 & 23 & 0.24 & 3.55 \\
\hline & 9360 & 9367.2 & 20 & 1.33 & 1.92 & 9360 & 9360.0 & 21 & 0.38 & 3.52 \\
\hline \multirow{3}{*}{ KroB 150} & 11260 & 11299.8 & 29 & 1.41 & 1.73 & 11225 & 11231.6 & 30 & 0.94 & 4.06 \\
\hline & 10121 & 10121.8 & 24 & 0.81 & 1.34 & 10121 & 10121.0 & 24 & 0.41 & 3.54 \\
\hline & 9542 & 9562.8 & 20 & 0.79 & 1.81 & 9542 & 9542.0 & 20 & 0.20 & 3.84 \\
\hline \multirow{3}{*}{ KroA 200} & 13042 & 13085.4 & 34 & 1.30 & 2.71 & 13041 & 13120.4 & 32 & 1.69 & 5.38 \\
\hline & 11392 & 11405.4 & 28 & 1.31 & 1.98 & 11392 & 11403.0 & 27 & 0.86 & 4.91 \\
\hline & 10535 & 10535.6 & 26 & 1.03 & 1.68 & 10528 & 10838.2 & 26 & 0.75 & 4.65 \\
\hline & 13022 & 13078.4 & 36 & 2.09 & 2.86 & 13020 & 13304.2 & 34 & 0.46 & 5.45 \\
\hline KroB 200 & 11713 & 11793.4 & 30 & 1.35 & 2.22 & 11712 & 11850.6 & 28 & 1.21 & 5.34 \\
\hline & 10619 & 10563.8 & 30 & 1.22 & 1.82 & 10614 & 10679.8 & 28 & 1.00 & 4.74 \\
\hline Avg & 8912.7 & 8927.4 & 17 & 0.47 & 1.27 & 8911.6 & 8932.2 & 16 & 0.29 & 2.54 \\
\hline No.Best & 40 & & & & & 48 & & & & \\
\hline
\end{tabular}


Table 8. Comparison of LS1 and LS2 on large binary GCSP instances with varying node costs

\begin{tabular}{|c|c|c|c|c|c|c|c|c|c|c|c|}
\hline \multirow{2}{*}{ Instance } & \multirow{2}{*}{ Range } & \multicolumn{5}{|c|}{ LS 1} & \multicolumn{5}{|c|}{ LS2 } \\
\hline & & $\begin{array}{l}\text { Best } \\
\text { Cost }\end{array}$ & $\begin{array}{l}\text { Avg. } \\
\text { Cost }\end{array}$ & NB & $\begin{array}{c}\text { Avg. } \\
\text { TB }\end{array}$ & $\begin{array}{c}\text { Avg. } \\
\text { TT }\end{array}$ & $\begin{array}{l}\text { Best } \\
\text { Cost }\end{array}$ & $\begin{array}{l}\text { Avg. } \\
\text { Cost }\end{array}$ & NB & $\begin{array}{c}\text { Avg. } \\
\text { TB }\end{array}$ & $\begin{array}{c}\text { Avg. } \\
\text { TT }\end{array}$ \\
\hline \multirow{3}{*}{ ts 225} & A & 169425 & 170633.6 & 65 & 11.7 & 17.8 & 169607 & 170402.8 & 64 & 1.7 & 6.0 \\
\hline & B & 81470 & 81674.4 & 66 & 9.3 & 13.5 & 80844 & 82654.0 & 66 & 2.1 & 6.8 \\
\hline & $\mathrm{C}$ & 72493 & 73388.8 & 67 & 9.8 & 13.5 & 72343 & 73266.4 & 70 & 1.4 & 6.6 \\
\hline \multirow{3}{*}{ a 280} & A & 3497 & 3508.6 & 76 & 19.4 & 27.2 & 3456 & 3467.8 & 78 & 3.8 & 8.6 \\
\hline & B & 1519 & 1538.0 & 79 & 12.2 & 22.9 & 1505 & 1533.6 & 81 & 5.8 & 9.1 \\
\hline & $\mathrm{C}$ & 1237 & 1251.6 & 83 & 17.4 & 22.9 & 1225 & 1247.8 & 84 & 3.2 & 8.5 \\
\hline \multirow{3}{*}{ pr299 } & A & 69521 & 69870.6 & 85 & 25.4 & 28.7 & 68958 & 69190.4 & 84 & 5.6 & 9.2 \\
\hline & B & 29871 & 30193.6 & 85 & 19.1 & 26.4 & 29855 & 30216.6 & 82 & 4.0 & 9.7 \\
\hline & $\mathrm{C}$ & 25100 & 25313.2 & 85 & 19.7 & 26.5 & 25010 & 25340.6 & 87 & 5.3 & 9.4 \\
\hline \multirow{3}{*}{$\operatorname{lin} 318$} & A & 68151 & 68465.8 & 90 & 20.0 & 36.7 & 67288 & 67962.2 & 89 & 2.6 & 9.5 \\
\hline & B & 27839 & 27885.4 & 90 & 19.1 & 32.2 & 27965 & 28214.8 & 93 & 4.3 & 9.6 \\
\hline & $\mathrm{C}$ & 22600 & 22790.4 & 95 & 24.6 & 31.5 & 22527 & 22786.6 & 96 & 3.6 & 9.8 \\
\hline \multirow{3}{*}{$\operatorname{pr} 439$} & $\mathrm{~A}$ & 232359 & 233265.0 & 124 & 58.7 & 87.0 & 228721 & 229267.4 & 127 & 7.3 & 15.3 \\
\hline & B & 82288 & 82658.6 & 128 & 41.9 & 83.5 & 81039 & 81303.4 & 128 & 6.9 & 15.0 \\
\hline & $\mathrm{C}$ & 62084 & 62604.8 & 134 & 59.7 & 83.0 & 61908 & 62719.0 & 133 & 10.1 & 15.2 \\
\hline \multirow{3}{*}{ att532 } & $\mathrm{A}$ & 170276 & 172554.8 & 142 & 86.1 & 150.9 & 169357 & 169963.0 & 143 & 8.0 & 19.0 \\
\hline & $\mathrm{B}$ & 57937 & 58263.4 & 147 & 110.8 & 142.5 & 56956 & 57560.4 & 145 & 16.3 & 19.8 \\
\hline & $\mathrm{C}$ & 43237 & 43709.6 & 150 & 106.0 & 149.0 & 43124 & 43582.4 & 149 & 9.5 & 20.5 \\
\hline \multirow{3}{*}{ ali535 } & A & 4703 & 4754.8 & 145 & 92.6 & 164.0 & 4684 & 4694.0 & 145 & 10.5 & 20.3 \\
\hline & B & 1546 & 1551.0 & 145 & 113.7 & 148.7 & 1522 & 1528.6 & 145 & 9.3 & 18.6 \\
\hline & $\mathrm{C}$ & 1084 & 1136.4 & 152 & 112.8 & 149.0 & 1082 & 1094.8 & 158 & 10.4 & 17.8 \\
\hline \multirow{3}{*}{ u574 } & A & 84623 & 85052.2 & 168 & 124.8 & 210.5 & 83652 & 83767.2 & 168 & 12.1 & 23.0 \\
\hline & $\mathrm{B}$ & 27148 & 27224.0 & 171 & 108.6 & 190.2 & 26792 & 26852.6 & 176 & 14.0 & 23.0 \\
\hline & $\mathrm{C}$ & 19448 & 19718.2 & 171 & 159.8 & 189.4 & 19227 & 19379.2 & 177 & 16.7 & 23.0 \\
\hline \multirow{3}{*}{ rat575 } & $\mathrm{A}$ & 13016 & 13115.4 & 161 & 136.0 & 210.4 & 12887 & 12946.0 & 164 & 12.5 & 22.9 \\
\hline & $\mathrm{B}$ & 4139 & 4198.2 & 159 & 142.7 & 189.1 & 4119 & 4152.6 & 158 & 16.7 & 21.8 \\
\hline & $\mathrm{C}$ & 2953 & 3015.4 & 162 & 175.7 & 185.0 & 2935 & 3015.6 & 175 & 17.4 & 22.1 \\
\hline \multirow{3}{*}{ p654 } & A & 114755 & 115350.0 & 180 & 174.2 & 306.9 & 113355 & 113694.0 & 182 & 21.3 & 30.8 \\
\hline & $\mathrm{B}$ & 34146 & 34212.0 & 184 & 171.3 & 286.9 & 33723 & 33795.8 & 182 & 17.0 & 27.8 \\
\hline & $\mathrm{C}$ & 25141 & 25184.8 & 193 & 129.7 & 282.3 & 24858 & 25170.8 & 195 & 14.4 & 25.5 \\
\hline \multirow{3}{*}{$\mathrm{d} 657$} & $\mathrm{~A}$ & 125916 & 127438.6 & 184 & 154.8 & 297.9 & 125211 & 125343.8 & 184 & 13.4 & 27.5 \\
\hline & $\mathrm{B}$ & 37816 & 38356.8 & 181 & 90.9 & 277.7 & 37434 & 37926.6 & 184 & 19.7 & 26.9 \\
\hline & $\mathrm{C}$ & 26378 & 26771.8 & 195 & 217.8 & 273.1 & 26209 & 26644.2 & 188 & 19.7 & 27.6 \\
\hline \multirow{3}{*}{ gr666 } & A & 8338 & 8482.8 & 187 & 199.5 & 317.9 & 8324 & 8339.0 & 186 & 13.1 & 28.1 \\
\hline & B & 2527 & 2537.8 & 187 & 273.3 & 314.9 & 2492 & 2495.6 & 189 & 20.3 & 25.0 \\
\hline & $\mathrm{C}$ & 1679 & 1691.8 & 194 & 264.2 & 289.7 & 1653 & 1675.4 & 199 & 14.9 & 25.5 \\
\hline \multirow{3}{*}{ u724 } & A & 101048 & 102655.0 & 197 & 92.3 & 397.2 & 100284 & 100547.0 & 202 & 12.5 & 30.8 \\
\hline & $\mathrm{B}$ & 29358 & 29534.0 & 196 & 119.0 & 384.2 & 29099 & 29310.8 & 198 & 20.3 & 30.2 \\
\hline & $\mathrm{C}$ & 20203 & 20788.2 & 197 & 299.9 & 362.0 & 20163 & 20558.4 & 210 & 14.7 & 31.0 \\
\hline \multirow{3}{*}{ rat783 } & A & 21450 & 21699.6 & 211 & 398.5 & 518.5 & 21272 & 21307.6 & 213 & 15.6 & 35.2 \\
\hline & $\mathrm{B}$ & 6085 & 6127.0 & 213 & 401.5 & 504.1 & 6034 & 6051.0 & 216 & 26.6 & 33.5 \\
\hline & $\mathrm{C}$ & 4109 & 4160.6 & 218 & 320.0 & 479.9 & 4054 & 4089.4 & 229 & 20.2 & 33.4 \\
\hline \multirow{3}{*}{ dsj 1000} & A & 68089542 & 68442158.0 & 280 & 580.1 & 693.3 & 67653673 & 67769758.6 & 283 & 49.6 & 72.8 \\
\hline & B & 16728234 & 16954235.0 & 281 & 500.1 & 680.2 & 16525509 & 16629826.4 & 285 & 45.8 & 56.1 \\
\hline & $\mathrm{C}$ & 10384875 & 10518746.0 & 292 & 413.5 & 605.5 & 10265949 & 10376224.8 & 295 & 47.5 & 57.5 \\
\hline Avg. & & 2158692.5 & 2174877.0 & 155 & 147.5 & 220.1 & 2141508.5 & 2149130.4 & 157 & 13.95 & 22.78 \\
\hline No. Best & & 2 & & & & & 43 & & & & \\
\hline
\end{tabular}


Table 9: Comparison of LS1 and LS2 on large GCSP without overnight instances with varying node costs

\begin{tabular}{|c|c|c|c|c|c|c|c|c|c|c|c|c|c|}
\hline \multirow[b]{2}{*}{ Instance } & \multirow[b]{2}{*}{ Range } & \multicolumn{6}{|c|}{ LS1 } & \multicolumn{6}{|c|}{ LS2 } \\
\hline & & $\begin{array}{l}\text { Best } \\
\text { Cost }\end{array}$ & $\begin{array}{l}\text { Avg. } \\
\text { Cost }\end{array}$ & NB & NR & $\begin{array}{c}\text { Avg. } \\
\text { TB }\end{array}$ & $\begin{array}{c}\text { Avg. } \\
\text { TT }\end{array}$ & $\begin{array}{l}\text { Best } \\
\text { Cost }\end{array}$ & $\begin{array}{l}\text { Avg. } \\
\text { Cost }\end{array}$ & NB & NR & $\begin{array}{c}\text { Avg. } \\
\text { TB }\end{array}$ & $\begin{array}{l}\text { Avg. } \\
\text { TT }\end{array}$ \\
\hline \multirow{3}{*}{ ts 225} & A & 170859 & 171026.8 & 64 & 4 & 15.0 & 27.8 & 169887 & 170929.8 & 64 & 4 & 7.8 & 17.7 \\
\hline & B & 83290 & 83457.4 & 66 & 0 & 11.4 & 25.4 & 83016 & 84221.8 & 64 & 2 & 8.6 & 18.2 \\
\hline & $\mathrm{C}$ & 72322 & 73054.4 & 67 & 0 & 15.0 & 25.6 & 72242 & 74070.0 & 68 & 1 & 19.8 & 19.4 \\
\hline \multirow{3}{*}{ a280 } & A & 3532 & 3561.6 & 77 & 4 & 33.5 & 45.6 & 3497 & 3525.0 & 79 & 5 & 7.8 & 24.1 \\
\hline & B & 1541 & 1567 & 78 & 0 & 27.2 & 41.1 & 1533 & 1552.2 & 78 & 1 & 16.2 & 25.4 \\
\hline & $\mathrm{C}$ & 1242 & 1266.2 & 84 & 0 & 34.2 & 42.2 & 1225 & 1274.8 & 89 & 1 & 20.4 & 24.0 \\
\hline \multirow{3}{*}{ pr299 } & A & 69803 & 70228.4 & 83 & 4 & 38.2 & 51.5 & 69084 & 69451.2 & 36 & 7 & 5.6 & 24.0 \\
\hline & B & 30541 & 30714 & 83 & 1 & 27.1 & 46.9 & 29975 & 30442.0 & 85 & 1 & 6.7 & 26.3 \\
\hline & $\mathrm{C}$ & 25320 & 25545.4 & 83 & 0 & 33.1 & 46.1 & 25211 & 25471.4 & 87 & 1 & 11.0 & 27.4 \\
\hline \multirow{3}{*}{$\operatorname{lin} 318$} & A & 64081 & 64541 & 92 & 12 & 54.6 & 72.5 & 63820 & 64043.4 & 93 & 12 & 10.4 & 27.4 \\
\hline & B & 27315 & 27499.6 & 92 & 5 & 33.1 & 56.5 & 27109 & 27449.2 & 98 & 14 & 12.6 & 26.9 \\
\hline & $\mathrm{C}$ & 22481 & 22637.6 & 98 & 5 & 48.1 & 56.0 & 22303 & 22441.8 & 99 & 9 & 19.5 & 28.2 \\
\hline \multirow{3}{*}{ pr439 } & A & 216792 & 217664.41 & 128 & 18 & 125.3 & 171.1 & 215284 & 215969.2 & 130 & 23 & 24.6 & 41.6 \\
\hline & $\mathrm{B}$ & 79204 & 80295.4 & 129 & 5 & 112.3 & 159.1 & 78779 & 79161.8 & 129 & 11 & 23.0 & 40.4 \\
\hline & $\mathrm{C}$ & 61520 & 62296.6 & 128 & 2 & 122.0 & 156.3 & 60607 & 61076.4 & 134 & 5 & 22.6 & 43.1 \\
\hline \multirow{3}{*}{ att532 } & A & 169494 & 170010.41 & 142 & 18 & 221.3 & 303.1 & 166189 & 166651.2 & 144 & 12 & 25.3 & 53.8 \\
\hline & $\mathrm{B}$ & 57427 & 57859.2 & 144 & 3 & 223.5 & 278.4 & 57411 & 57996.2 & 148 & 4 & 41.2 & 50.6 \\
\hline & $\mathrm{C}$ & 42995 & 43717.8 & 146 & 3 & 218.2 & 282.5 & 42551 & 43167.2 & 156 & 6 & 26.5 & 53.0 \\
\hline \multirow{3}{*}{ ali535 } & A & 4432 & 4456.6 & 144 & 24 & 248.4 & 319.1 & 4396 & 4431.0 & 147 & 30 & 76.0 & 76.0 \\
\hline & B & 1527 & 1534.4 & 142 & 4 & 252.2 & 279.5 & 1503 & 1516.6 & 148 & 10 & 12.2 & 46.2 \\
\hline & $\mathrm{C}$ & 1094 & 1107.6 & 153 & 10 & 202.6 & 261.2 & 1070 & 1084.6 & 204 & 35 & 39.8 & 50.1 \\
\hline \multirow{3}{*}{ u574 } & A & 81485 & 82068.6 & 170 & 22 & 434.0 & 455.4 & 80016 & 80217.4 & 174 & 33 & 16.3 & 59.8 \\
\hline & $\mathrm{B}$ & 26466 & 26618 & 169 & 8 & 329.5 & 398.8 & 26305 & 26461.4 & 177 & 9 & 40.0 & 60.7 \\
\hline & $\mathrm{C}$ & 18885 & 19537.8 & 175 & 5 & 254.9 & 393.7 & 18794 & 19002.8 & 183 & 13 & 61.1 & 63.0 \\
\hline \multirow{3}{*}{ rat575 } & A & 12683 & 12735 & 160 & 16 & 360.4 & 437.0 & 12330 & 12365.8 & 164 & 24 & 40.1 & 56.2 \\
\hline & B & 4109 & 4145.2 & 162 & 3 & 352.9 & 391.4 & 4086 & 4119.0 & 162 & 2 & 35.3 & 55.5 \\
\hline & $\mathrm{C}$ & 2993 & 3026.8 & 164 & 0 & 265.9 & 395.0 & 2927 & 2966.4 & 169 & 1 & 44.0 & 55.5 \\
\hline \multirow{3}{*}{ p654 } & $\mathrm{A}$ & 110156 & 110240.8 & 187 & 31 & 715.4 & 888.4 & 107189 & 107689.4 & 187 & 43 & 98.6 & 104.9 \\
\hline & $\mathrm{B}$ & 33702 & 33942.2 & 179 & 9 & 466.6 & 631.7 & 33663 & 33861.0 & 187 & 12 & 54.8 & 72.8 \\
\hline & $\mathrm{C}$ & 24260 & 24629.2 & 194 & 6 & 517.2 & 646.7 & 24680 & 24780.8 & 199 & 2 & 58.7 & 69.8 \\
\hline \multirow{3}{*}{ d657 } & A & 122409 & 123344.4 & 181 & 27 & 542.5 & 631.4 & 118956 & 119302.0 & 185 & 39 & 47.1 & 68.1 \\
\hline & B & 37647 & 37867.8 & 182 & 2 & 529.8 & 602.1 & 37078 & 37505.2 & 184 & 1 & 41.4 & 68.9 \\
\hline & $\mathrm{C}$ & 26544 & 27463 & 184 & 1 & 546.4 & 570.2 & 26177 & 26506.2 & 194 & 5 & 41.6 & 70.4 \\
\hline \multirow{3}{*}{ gr666 } & A & 7999 & 8037.2 & 185 & 25 & 594.5 & 689.0 & 7805 & 7872.4 & 193 & 27 & 22.6 & 69.1 \\
\hline & $\mathrm{B}$ & 2418 & 2447.8 & 188 & 11 & 566.8 & 670.9 & 2417 & 2434.8 & 190 & 10 & 46.5 & 64.5 \\
\hline & $\mathrm{C}$ & 1660 & 1701.8 & 194 & 8 & 288.9 & 600.1 & 1636 & 1650.8 & 220 & 11 & 42.5 & 63.6 \\
\hline \multirow{3}{*}{ u724 } & A & 102454 & 102945.8 & 204 & 27 & 822.1 & 929.2 & 100132 & 100690.0 & 205 & 30 & 33.9 & 79.0 \\
\hline & $\mathrm{B}$ & 29643 & 29909.4 & 200 & 2 & 699.8 & 856.2 & 29257 & 29582.2 & 202 & 6 & 41.3 & 74.9 \\
\hline & $\mathrm{C}$ & 20542 & 20633.2 & 203 & 5 & 558.5 & 809.9 & 20390 & 20656.0 & 208 & 8 & 61.6 & 77.4 \\
\hline \multirow{3}{*}{ rat783 } & A & 20623 & 20805.8 & 192 & 29 & 924.9 & 1057.1 & 20150 & 20208.4 & 214 & 30 & 41.8 & 84.4 \\
\hline & B & 6013 & 6084 & 212 & 8 & 814.2 & 1015.8 & 5905 & 5965.0 & 215 & 8 & 61.3 & 81.7 \\
\hline & $\mathrm{C}$ & 4128 & 4153.4 & 225 & 5 & 719.0 & 950.5 & 4030 & 4053.8 & 229 & 4 & 57.9 & 82.8 \\
\hline & A & 64021453 & 64572154.2 & 276 & 58 & 1004.1 & 1378.4 & 63550808 & 63803980.8 & 288 & 61 & 206.2 & 235.1 \\
\hline dsj1000 & $\mathrm{B}$ & 16801430 & 17051243.6 & 281 & 11 & 924.7 & 1242.3 & 16500781 & 16546597.2 & 278 & 12 & 96.3 & 145.5 \\
\hline & $\mathrm{C}$ & 10524851 & 10689214 & 301 & 6 & 820.2 & 1151.3 & 10395420 & 10493207.0 & 299 & 7 & 111.8 & 137.2 \\
\hline Average & & 2072252.6 & 2093977.6 & 155 & 10 & 358.9 & 456.4 & 2051725.0 & 2060835.6 & 160 & 13 & 40.9 & 61.0 \\
\hline No. Best & & 1 & & & & & & 44 & & & & & \\
\hline
\end{tabular}


Table 10. Comparison of LS1 and LS2 on large GCSP with overnight instances with varying node costs

\begin{tabular}{|c|c|c|c|c|c|c|c|c|c|c|c|c|c|}
\hline \multirow[b]{2}{*}{ Instance } & \multirow[b]{2}{*}{ Range } & \multicolumn{6}{|c|}{ LS1 } & \multicolumn{6}{|c|}{ LS2 } \\
\hline & & $\begin{array}{l}\text { Best } \\
\text { Cost }\end{array}$ & $\begin{array}{l}\text { Avg. } \\
\text { Cost }\end{array}$ & NB & NR & $\begin{array}{c}\text { Avg. } \\
\text { TB }\end{array}$ & $\begin{array}{c}\text { Avg. } \\
\text { TT }\end{array}$ & $\begin{array}{l}\text { Best } \\
\text { Cost }\end{array}$ & $\begin{array}{l}\text { Avg. } \\
\text { Cost }\end{array}$ & NB & NR & $\begin{array}{c}\text { Avg. } \\
\text { TB }\end{array}$ & $\begin{array}{c}\text { Avg. } \\
\text { TT }\end{array}$ \\
\hline \multirow{3}{*}{ ts 225} & A & 144953 & 145012.4 & 67 & 28 & 16.1 & 24.2 & 143495 & 145716.8 & 71 & 37 & 2.3 & 15.0 \\
\hline & $\mathrm{B}$ & 73412 & 75075.4 & 74 & 38 & 17.3 & 21.9 & 73285 & 74478.0 & 71 & 37 & 7.6 & 16.2 \\
\hline & $\mathrm{C}$ & 63552 & 66184.2 & 76 & 44 & 15.1 & 23.5 & 63075 & 64390.8 & 73 & 43 & 7.9 & 16.3 \\
\hline \multirow{3}{*}{ a280 } & A & 2839 & 2869.2 & 84 & 31 & 34.8 & 42.6 & 2817 & 2825.2 & 88 & 46 & 2.5 & 20.1 \\
\hline & B & 1335 & 1369.8 & 81 & 28 & 22.9 & 32.4 & 1329 & 1336.4 & 89 & 43 & 6.6 & 21.8 \\
\hline & $\mathrm{C}$ & 1091 & 1109.6 & 87 & 31 & 20.8 & 28.8 & 1072 & 1093.0 & 89 & 32 & 10.0 & 22.8 \\
\hline \multirow{3}{*}{ pr299 } & $\mathrm{A}$ & 56842 & 57183.0 & 87 & 32 & 28.4 & 47.9 & 56760 & 57019.2 & 93 & 47 & 12.8 & 21.8 \\
\hline & $\mathrm{B}$ & 25937 & 26078.2 & 93 & 42 & 33.6 & 41.3 & 25774 & 26478.6 & 97 & 52 & 11.6 & 23.6 \\
\hline & $\mathrm{C}$ & 22301 & 22069.0 & 90 & 43 & 28.4 & 39.1 & 22018 & 22249.4 & 93 & 50 & 18.1 & 25.1 \\
\hline \multirow{3}{*}{$\operatorname{lin} 318$} & $\mathrm{~A}$ & 55557 & 55687.2 & 94 & 37 & 46.6 & 58.1 & 55327 & 55521.8 & 99 & 49 & 3.8 & 22.6 \\
\hline & $\mathrm{B}$ & 23772 & 24193.0 & 97 & 49 & 31.9 & 53.3 & 23431 & 23785.4 & 100 & 56 & 8.9 & 24.1 \\
\hline & $\mathrm{C}$ & 19844 & 20040.2 & 96 & 50 & 41.9 & 55.8 & 19471 & 19933.6 & 101 & 58 & 13.8 & 25.4 \\
\hline \multirow{3}{*}{ pr439 } & A & 184831 & 185494.4 & 128 & 43 & 131.3 & 170.5 & 183619 & 184851.6 & 138 & 70 & 17.0 & 36.5 \\
\hline & $\mathrm{B}$ & 69547 & 71507.0 & 130 & 57 & 114.9 & 145.7 & 68774 & 69588.2 & 140 & 75 & 25.0 & 39.6 \\
\hline & $\mathrm{C}$ & 54641 & 56134.0 & 133 & 63 & 97.7 & 143.4 & 54102 & 55320.0 & 144 & 81 & 33.8 & 45.4 \\
\hline \multirow{3}{*}{ att532 } & $\mathrm{A}$ & 136371 & 137644.0 & 147 & 45 & 185.1 & 226.2 & 135280 & 136056.2 & 150 & 54 & 30.8 & 47.1 \\
\hline & $\mathrm{B}$ & 48943 & 49748.6 & 152 & 60 & 160.0 & 197.1 & 48098 & 48542.6 & 158 & 81 & 21.8 & 46.7 \\
\hline & $\mathrm{C}$ & 37104 & 37892.2 & 161 & 68 & 133.8 & 186.0 & 37245 & 37551.4 & 170 & 100 & 43.8 & 53.4 \\
\hline \multirow{3}{*}{ ali535 } & $\mathrm{A}$ & 3714 & 3848.6 & 144 & 39 & 150.3 & 226.4 & 3672 & 3680.6 & 154 & 64 & 19.6 & 45.3 \\
\hline & $\mathrm{B}$ & 1338 & 1373.8 & 144 & 38 & 109.3 & 189.3 & 1321 & 1337.0 & 157 & 60 & 22.9 & 44.1 \\
\hline & $\mathrm{C}$ & 987 & 1005.8 & 152 & 25 & 119.6 & 166.5 & 979 & 996.0 & 162 & 32 & 42.9 & 50.7 \\
\hline \multirow{3}{*}{ u574 } & $\mathrm{A}$ & 64242 & 64451.8 & 171 & 64 & 195.8 & 322.7 & 63405 & 63747.6 & 183 & 89 & 15.3 & 49.8 \\
\hline & $\mathrm{B}$ & 21935 & 23033.4 & 169 & 67 & 245.8 & 275.7 & 21744 & 21913.8 & 191 & 102 & 48.0 & 59.2 \\
\hline & $\mathrm{C}$ & 16745 & 17193.8 & 179 & 86 & 184.9 & 265.8 & 16441 & 16638.2 & 190 & 108 & 58.6 & 62.5 \\
\hline \multirow{3}{*}{ rat575 } & A & 10295 & 10392.4 & 161 & 46 & 218.9 & 297.5 & 10196 & 10239.2 & 180 & 82 & 30.0 & 53.3 \\
\hline & $\mathrm{B}$ & 3508 & 3666.0 & 161 & 52 & 177.9 & 261.2 & 3465 & 3521.8 & 175 & 83 & 43.9 & 55.2 \\
\hline & $\mathrm{C}$ & 2663 & 2707.6 & 175 & 64 & 169.3 & 244.4 & 2600 & 2619.4 & 194 & 96 & 44.5 & 58.9 \\
\hline \multirow{3}{*}{ p654 } & A & 95019 & 100537.4 & 186 & 50 & 361.7 & 494.2 & 94169 & 94400.8 & 194 & 76 & 61.5 & 73.2 \\
\hline & $\mathrm{B}$ & 30053 & 30539.6 & 190 & 59 & 170.3 & 438.0 & 29718 & 29850.0 & 198 & 92 & 45.1 & 69.2 \\
\hline & $\mathrm{C}$ & 22965 & 23036.8 & 193 & 65 & 248.2 & 399.4 & 22566 & 22681.0 & 215 & 109 & 66.3 & 77.5 \\
\hline \multirow{3}{*}{ d657 } & $\mathrm{A}$ & 97053 & 98678.0 & 181 & 58 & 301.5 & 424.1 & 96632 & 96926.6 & 189 & 84 & 46.9 & 66.4 \\
\hline & $\mathrm{B}$ & 31322 & 33283.4 & 182 & 74 & 207.4 & 387.6 & 31153 & 31503.0 & 200 & 109 & 53.0 & 69.0 \\
\hline & $\mathrm{C}$ & 23308 & 24737.2 & 184 & 75 & 258.8 & 375.4 & 23120 & 23479.4 & 218 & 120 & 65.8 & 75.1 \\
\hline \multirow{3}{*}{ gr666 } & $\mathrm{A}$ & 6601 & 6771.0 & 189 & 49 & 292.6 & 436.9 & 6505 & 6524.8 & 206 & 89 & 36.6 & 68.3 \\
\hline & $\mathrm{B}$ & 2128 & 2148.2 & 194 & 64 & 278.7 & 417.0 & 2088 & 2093.6 & 208 & 95 & 43.1 & 64.8 \\
\hline & $\mathrm{C}$ & 1492 & 1533.4 & 198 & 41 & 262.8 & 353.2 & 1465 & 1492.4 & 227 & 65 & 45.2 & 72.7 \\
\hline \multirow{3}{*}{$\mathrm{u} 724$} & A & 81276 & 81851.8 & 204 & 57 & 339.3 & 568.1 & 81017 & 81290.0 & 216 & 93 & 41.1 & 76.5 \\
\hline & $\mathrm{B}$ & 25274 & 26464.0 & 198 & 62 & 186.3 & 496.5 & 24932 & 25082.4 & 228 & 117 & 50.3 & 72.3 \\
\hline & $\mathrm{C}$ & 18289 & 19011.8 & 209 & 91 & 378.1 & 494.0 & 18057 & 18113.2 & 234 & 138 & 79.2 & 85.5 \\
\hline \multirow{3}{*}{ rat783 } & A & 17337 & 17432.2 & 215 & 48 & 382.5 & 699.8 & 17222 & 17303.6 & 222 & 81 & 64.6 & 88.7 \\
\hline & $\mathrm{B}$ & 5130 & 5168.8 & 213 & 56 & 362.6 & 642.4 & 5106 & 5159.0 & 241 & 112 & 48.1 & 83.7 \\
\hline & $\mathrm{C}$ & 3650 & 3833.4 & 228 & 77 & 486.3 & 619.9 & 3604 & 3623.0 & 247 & 116 & 67.4 & 84.8 \\
\hline & A & 53016230 & 52924952.8 & 293 & 101 & 412.6 & 823.6 & 52767955 & 52811295.2 & 302 & 122 & 138.6 & 175.2 \\
\hline dsj1000 & $\mathrm{B}$ & 13904578 & 14157851.0 & 305 & 101 & 500.5 & 803.5 & 13714835 & 13832973.2 & 309 & 151 & 115.8 & 154.1 \\
\hline & $\mathrm{C}$ & 8992587 & 9038746.0 & 301 & 83 & 401.5 & 785.9 & 8946964 & 9010501.0 & 309 & 169 & 179.1 & 181.0 \\
\hline Average & & 1722724.2 & 1727989.8 & 160 & 55 & 190.3 & 298.8 & 1711686.7 & 1717016.09 & 171 & 81 & 41.1 & 58.7 \\
\hline No. Best & & 1 & & & & & & 44 & & & & & \\
\hline
\end{tabular}


Table 11. Comparison of computing times of GTSP methods

\begin{tabular}{|c|c|c|c|}
\hline Computer & Mflops & $\mathrm{r}$ & Method \\
\hline Gateway Profile 4MX & 230 & 1.568 & GA \\
\hline Sun Sparc Station LX & 4.6 & 0.032 & GI $^{3}$, NN \\
\hline HP 9000/720 & 2.3 & 0.016 & FST-Lagr, FST-Root, B\&C \\
\hline Unknown & - & 1 & RACS \\
\hline Dell Dimension 8400 & 290 & 2 & MSAX \\
\hline Pentium(R) IV, 3.4 Ghz & 295 & 2.03 & LS2 \\
\hline Ours & 145 & 1 & \\
\hline
\end{tabular}


Table 12. Comparison of LS2 against 8 other heuristics on benchmark GTSP instances in the literature

\begin{tabular}{|c|c|c|c|c|c|c|c|c|c|c|c|c|c|c|c|c|c|c|}
\hline \multirow{2}{*}{ Instances } & \multicolumn{2}{|c|}{ LS2 } & \multicolumn{2}{|c|}{ MSA } & \multicolumn{2}{|c|}{$\operatorname{mrOx}$} & \multirow{2}{*}{$\frac{\text { RACS }}{\text { gap }}$} & \multicolumn{2}{|c|}{ GA } & \multicolumn{2}{|c|}{$\mathrm{GI}^{3}$} & \multicolumn{2}{|c|}{ NN } & \multicolumn{2}{|c|}{ FST-lagr } & \multicolumn{2}{|c|}{ FST-Root } & \multirow{2}{*}{$\frac{B \& C}{\text { time }}$} \\
\hline & gap & time & gap & time & gap & time & & gap & time & gap & time & gap & time & gap & time & gap & time & \\
\hline Att48 & 0 & 0.4 & 0 & 0 & 0 & 0.8 & - & 0 & 0 & - & - & - & - & 0 & 0 & 0 & 0 & 0.0 \\
\hline Gr48 & 0 & 0.4 & 0 & 0 & 0 & 0.6 & - & 0 & 0.8 & - & - & - & - & 0 & 0 & 0 & 0 & 0.0 \\
\hline Hk48 & 0 & 0.5 & 0 & 0 & 0 & 0.6 & - & 0 & 0.4 & - & - & - & - & 0 & 0 & 0 & 0 & 0.0 \\
\hline Eil51 & 0 & 0.5 & 0 & 0 & 0 & 0.6 & 0 & 0 & 0.2 & 0 & 0 & 0 & 0 & 0 & 0 & 0 & 0 & 0.0 \\
\hline Brazil58 & 0 & 0.6 & 0 & 0 & 0 & 1.6 & - & 0 & 0.4 & - & - & - & - & 0 & 0 & 0 & 0 & 0.0 \\
\hline St70 & 0 & 0.7 & 0 & 0 & 0 & 0.8 & 0 & 0 & 0.4 & 0 & 0 & 0 & 0 & 0 & 0 & 0 & 0.2 & 0.2 \\
\hline Eil76 & 0 & 0.8 & 0 & 0 & 0 & 0.8 & 0 & 0 & 0.4 & 0 & 0 & 0 & 0 & 0 & 0 & 0 & 0.2 & 0.2 \\
\hline Pr76 & 0 & 0.9 & 0 & 0 & 0 & 1.0 & 0 & 0 & 0.4 & 0 & 0 & 0 & 0 & 0 & 0 & 0 & 0.2 & 0.2 \\
\hline Rat99 & 0 & 1.2 & 0 & 0 & 0 & 1.0 & 0 & 0 & 1.0 & 0 & 0.2 & 0 & 0.2 & 0 & 0 & 0 & 0.8 & 0.8 \\
\hline KroA100 & 0 & 1.2 & 0 & 0 & 0 & 1.2 & 0 & 0 & 0.6 & 0 & 0.2 & 0 & 0.2 & 0 & 0 & 0 & 0.2 & 0.2 \\
\hline KroB100 & 0 & 1.3 & 0 & 0 & 0 & 1.2 & 0 & 0 & 0.6 & 0 & 0.2 & 0 & 0 & 0 & 0 & 0 & 0.4 & 0.4 \\
\hline KroC100 & 0 & 1.2 & 0 & 0 & 0 & 1.2 & 0 & 0 & 0.4 & 0 & 0.2 & 0 & 0.2 & 0 & 0 & 0 & 0.2 & 0.2 \\
\hline KroD100 & 0 & 1.2 & 0 & 0 & 0 & 1.4 & 0 & 0 & 0.6 & 0 & 0.2 & 0 & 0. & 0 & 0 & 0 & 0.2 & 0.2 \\
\hline KroE100 & 0 & 1.3 & 0 & 0 & 0 & 1.2 & 0 & 0 & 1.2 & 0 & 0.2 & 0 & 0 & 0 & 0 & 0 & 0.2 & 0.2 \\
\hline Rd100 & 0 & 1.2 & 0 & 0 & 0 & 1.0 & 0 & 0 & 0.4 & 0.08 & 0.2 & 0.08 & 0.2 & 0.08 & 0 & 0 & 0.2 & 0.2 \\
\hline Eil101 & 0 & 1.1 & 0 & 0 & 0 & 1.0 & 0 & 0 & 0.4 & 0.4 & 0.2 & 0.4 & 0 & 0 & 0 & 0 & 0.4 & 0.4 \\
\hline Lin105 & 0 & 1.3 & 0 & 0 & 0 & 1.2 & 0 & 0 & 0.4 & 0 & 0.4 & 0 & 0.2 & 0 & 0 & 0 & 0.2 & 0.2 \\
\hline Pr107 & 0 & 1.2 & 0 & 0 & 0 & 1.0 & 0 & 0 & 0.6 & 0 & 0.2 & 0 & 0.2 & 0 & 0 & 0 & 0.2 & 0.2 \\
\hline Gr120 & 0 & 1.1 & 0 & 0 & 0 & 1.4 & - & 0 & 0.8 & - & - & - & - & 1.99 & 0 & 0 & 0.6 & 0.6 \\
\hline Pr124 & 0 & 1.5 & 0 & 0 & 0 & 1.4 & 0 & 0 & 1.0 & 0.43 & 0.4 & 0 & 0.4 & 0 & 0 & 0 & 0.4 & 0.4 \\
\hline Bier127 & 0.04 & 1.6 & 0 & 0 & 0 & 1.6 & 0 & 0 & 0.8 & 5.55 & 1.0 & 9.68 & 0.2 & 0 & 0.2 & 0 & 0.4 & 0.4 \\
\hline Pr136 & 0 & 1.8 & 0 & 0 & 0 & 1.6 & 0 & 0 & 0.8 & 1.28 & 0.4 & 5.54 & 0.2 & 0.82 & 0.2 & 0 & 0.6 & 0.6 \\
\hline Pr144 & 0 & 1.6 & 0 & 0 & 0 & 2.0 & 0 & 0 & 0.4 & 0 & 0.4 & 0 & 0.4 & 0 & 0 & 0 & 0.2 & 0.2 \\
\hline KroA150 & 0 & 2.1 & 0 & 0 & 0 & 2.0 & 0 & 0 & 2.0 & 0 & 0.6 & 0 & 0.6 & 0 & 0.2 & 0 & 1.4 & 1.5 \\
\hline KroB150 & 0 & 1.9 & 0 & 0 & 0 & 2.0 & 0 & 0 & 1.6 & 0 & 0.4 & 0 & 0.6 & 0 & 0.2 & 0 & 0.8 & 0.8 \\
\hline Pr152 & 0 & 2.0 & 0 & 0 & 0 & 2.0 & 0 & 0 & 2.4 & 0.47 & 0.6 & 1.8 & 0.4 & 0 & 0.2 & 0 & 0.8 & 1.5 \\
\hline
\end{tabular}




\begin{tabular}{|c|c|c|c|c|c|c|c|c|c|c|c|c|c|c|c|c|c|c|}
\hline \multirow{2}{*}{ Instances } & \multicolumn{2}{|c|}{ LS2 } & \multicolumn{2}{|c|}{ MSA } & \multicolumn{2}{|c|}{$\operatorname{mrOX}$} & RACS & \multicolumn{2}{|c|}{ GA } & \multicolumn{2}{|c|}{$\mathrm{GI}^{3}$} & \multicolumn{2}{|c|}{$\mathrm{NN}$} & \multicolumn{2}{|c|}{ FST-lagr } & \multicolumn{2}{|c|}{ FST-Root } & \multirow{2}{*}{$\frac{\mathrm{B} \& \mathrm{C}}{\text { time }}$} \\
\hline & gap & time & gap & time & gap & time & gap & gap & time & gap & time & gap & time & gap & time & gap & time & \\
\hline U159 & 0 & 2.2 & 0 & 0 & 0 & 2.0 & 0.01 & 0 & 1.0 & 2.6 & 0.6 & 2.79 & 0.8 & 0 & 0.2 & 0 & 2.0 & 2.0 \\
\hline Rat195 & 0 & 2.5 & 0 & 0.2 & 0 & 2.8 & 0 & 0 & 1.0 & 0 & 1.2 & 1.29 & 2.6 & 1.87 & 0.2 & 0 & 3.5 & 3.5 \\
\hline D198 & 0.32 & 3.4 & 0 & 0 & 0 & 3.2 & 0.01 & 0 & 1.8 & 0.6 & 1.8 & 0.6 & 3.6 & 0.48 & 0.2 & 0 & 10.8 & 10.8 \\
\hline KroA200 & 0 & 2.8 & 0 & 0 & 0 & 3.4 & 0.01 & 0 & 4.2 & 0 & 0.8 & 5.25 & 1.6 & 0 & 0.2 & 0 & 2.6 & 2.6 \\
\hline KroB200 & 0 & 2.7 & 0 & 0 & 0.05 & 3.2 & 0 & 0 & 2.2 & 0 & 1.0 & 0 & 4.0 & 0.05 & 0.2 & 0 & 3.9 & 3.9 \\
\hline Ts225 & 0 & 2.9 & 0 & 4.3 & 0.14 & 3.4 & 0.02 & 0 & 3.9 & 0.61 & 2.6 & 0 & 3.6 & 0.09 & 0.2 & 0.09 & 18.5 & 538.2 \\
\hline Pr226 & 0.09 & 2.5 & 0 & 0 & 0 & 3.0 & 0.03 & 0 & 1.6 & 0 & 0.8 & 2.17 & 2.0 & 0 & 0.2 & 0 & 1.4 & 1.4 \\
\hline Gil262 & 0.79 & 3.6 & 0 & 7.1 & 0.45 & 7.2 & 0.22 & 0.79 & 3.0 & 5.03 & 3.5 & 1.88 & 3.6 & 3.75 & 0.2 & 0.89 & 20.5 & 94.2 \\
\hline Pr264 & 0.59 & 3.6 & 0 & 0 & 0 & 4.8 & 0 & 0 & 2.0 & 0.36 & 2.0 & 5.73 & 4.5 & 0.33 & 0.4 & 0 & 4.9 & 4.9 \\
\hline Pr299 & 0.04 & 4.5 & 0 & 1.4 & 0.05 & 9.2 & 0.24 & 0.02 & 9.7 & 2.23 & 0.2 & 2.01 & 8.5 & 0 & 0.4 & 0 & 11.6 & 11.6 \\
\hline Lin318 & 0.01 & 4.4 & 0 & 0 & 0 & 16.2 & 0.12 & 0 & 5.5 & 4.59 & 6.2 & 4.92 & 9.7 & 0.36 & 0.8 & 0.36 & 12.0 & 23.8 \\
\hline Rd400 & 0.98 & 6.2 & 0 & 0.6 & 0.58 & 29.2 & 0.87 & 1.37 & 5.5 & 1.23 & 12.2 & 3.98 & 34.7 & 3.16 & 0.8 & 2.97 & 71.5 & 99.9 \\
\hline F1417 & 0.01 & 5.8 & 0 & 0 & 0.04 & 16.4 & 0.57 & 0.07 & 3.8 & 0.48 & 12.8 & 1.07 & 40.8 & 0.13 & 1.0 & 0 & 237.5 & 237.5 \\
\hline Pr439 & 0.09 & 7.1 & 0 & 3.9 & 0 & 38.2 & 0.79 & 0.23 & 14.4 & 3.52 & 18.4 & 4.02 & 37.8 & 1.42 & 2.0 & 0 & 76.9 & 77.1 \\
\hline Pcb442 & 0.16 & 6.9 & 0 & 1.6 & 0.01 & 46.8 & 0.69 & 1.31 & 16.0 & 5.91 & 17.0 & 0.22 & 25.6 & 4.22 & 1.2 & 0.29 & 76.1 & 835.1 \\
\hline Average (36) & 0.09 & 2.5 & 0 & 0.53 & 0.04 & 6.12 & 0.10 & 0.10 & 2.58 & 0.98 & 2.42 & 1.48 & 5.21 & 0.46 & 0.26 & 0.13 & 15.61 & 54.32 \\
\hline \# Opt (36) & \multicolumn{2}{|c|}{25} & \multicolumn{2}{|c|}{36} & \multicolumn{2}{|c|}{29} & 24 & \multicolumn{2}{|c|}{30} & \multicolumn{2}{|c|}{19} & \multicolumn{2}{|c|}{18} & \multicolumn{2}{|c|}{23} & \multicolumn{2}{|c|}{31} & 36 \\
\hline Average (41) & 0.08 & 2.2 & 0 & 0.47 & 0.03 & 5.38 & & 0.09 & 2.31 & & & & & 0.46 & 0.22 & 0.11 & 13.72 & 47.71 \\
\hline \# Opt (41) & \multicolumn{2}{|c|}{30} & \multicolumn{2}{|c|}{41} & \multicolumn{2}{|c|}{34} & & \multicolumn{2}{|c|}{35} & & & & & \multicolumn{2}{|c|}{27} & \multicolumn{2}{|c|}{36} & 41 \\
\hline
\end{tabular}

